\title{
MFASUREMENT OF REPUISTIVE FORCES
}

BETWETN CHARGED PHOSPHOLIPID BILAYERS.

$$
\text { A.C. Cowley Basc., PhD. }
$$

Department of Biological Sciences

Thesis submitted in partial fulfillment of the requirements for the degree of Master of Science.

Brock University

St.Catharines, Ontario. November 1979.

(c) A. C. Cowley, 1980 


\section{ABSTRACT.}

Electrostatic forces between membranes containing charged lipids were assumed to play an important role in influencing interactions between membranes long before quantitative measurements of such forces were available. dur measurements were designed to measure electrostatic forces between layers of lecithin charged with lipids carrying ionizable head groups. These experiments have shown that the interactions between charged lipid bilayers are dominated by electrostatic forces only at separations greater than $30 \mathrm{~A}$. At smaller separations the repulsion between charged bjlayers is dominated by strong hydration forces.

The net repulsive force between egg lecithin bilayers containing various amounts of charged lipids (phosphatidylglycerol ( $P G) 5,10$ and 50 mole\%, phosphatidylinositol (PI) $10 \mathrm{~mole} \%$ and sodium oleate ( $\mathrm{Na}-\mathrm{OI}$ ) 3,5 and 10 mole\%, where mole\% gives the ratio of the number of moles of charged linid to the total number of moles of all lipids present in the sample) was studied with the help of the osmotic stress technique described by LeNeveu et a. . (1977). AIso, the forces between pure $P G$ were investigated in the same manner. The results have been plotted showing variation of force as a function of bilayer separation $d_{w}$. All curves so obtained called force curves, were found to be similar in shape, showing two distinct regions, one when $a_{w}<30 \mathrm{~A}$ is a region of very rapid 
variation of force with separation ( it is the region dominated by hydration force) and second when $a_{\text {w }}>40 \mathrm{~A}$ is a region of very slow variation of force with separation ( it is the region dominated by the electrostatic force). Between these two regions there exists a transition area in which, in most systems studied, a phase separation of lipids into fractions containing different amounts of charged groups, was observed.

A qualitative analysis showed that our results were well described by the simple electrostatic double-layer theory. For quantitative agreement between measured and calculated force curves however, the charge density for the calculations had to be taken as half of that given by the number density of charged lipids present in the lecithin bilayers. It is not clear at the moment what causes such low apparent degree of ionization among the charged head groups, and further study is needed in this area. 


\section{ACKNOWTIFDGEMENTS.}

I wish to thank Dr. R.P. Rand for suggesting these experiments, for his inspiring guidence during this study and for his financial support.

I would also like to thank Dr. V.A. Parsegian for his invaluable discussions and sharing of his insights into the theory of interactions between macroscopic bodies.

Thanks are also extended to Mrs. Nola Fuller for her help in the experiments and her friendshjp.

I am also indebted to my mother for her help and to my husband for his support and interest in this work.

Finally I wish to thank the Department of Biological Sciences for offering me the working facilities and friendly surroundings. 


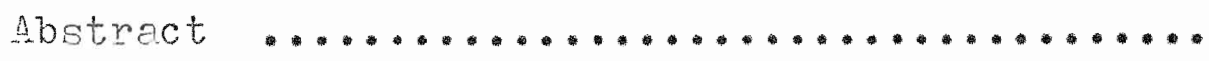

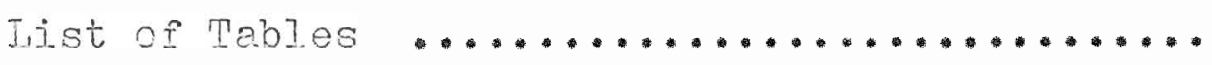

$\mathrm{V} \dot{i}$

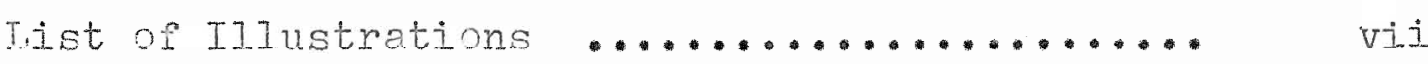

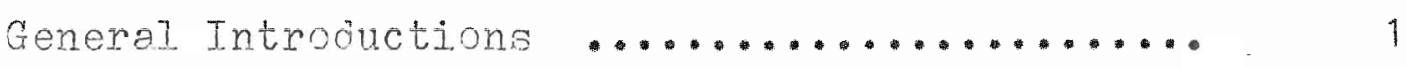

1. Materials and Methods .................... 22

1.1 Mixjing of charged linids with egg lecithin 22

1.2 Preparation of dextran solutions ..... 23

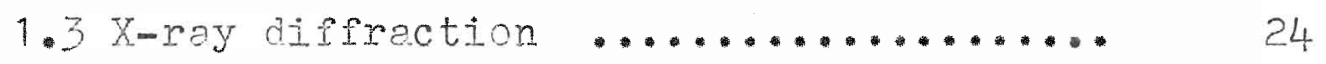

1.4 Prenaration of X-ray samples ....... 30

2. Results and Interpretation of Results .... 33

2.1 The Iipid-water phase diagrams ....... 33

2.2 Swelling of lipid against osmotic pres-

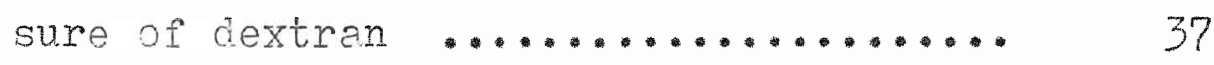

2.3 The force curves for different lipid

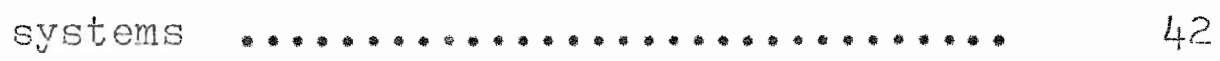

2.4 Rffects of salt solutions and $\mathrm{pH} \ldots 52$

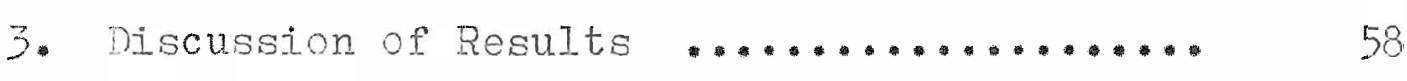

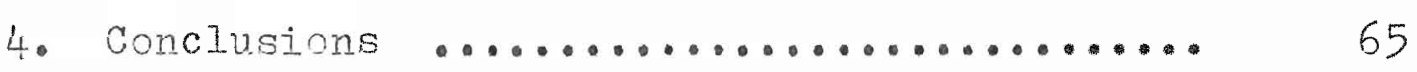

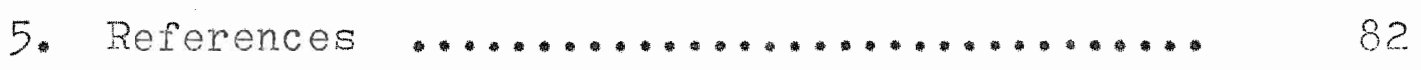
Appendix $T$

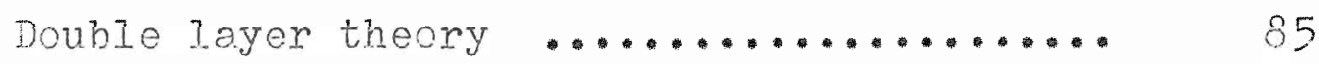
Appendix II

Calibration of the photographs with Teflon

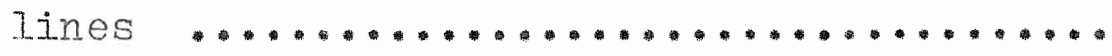




\section{LIST OF TABI,FS}

Table

I Conductance measurements for water and

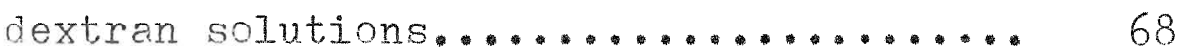

II Refractive indices of dextran solutions.. 69

II "d" spacings for Na-oleate lecithin system swelled in dextran solutions containing

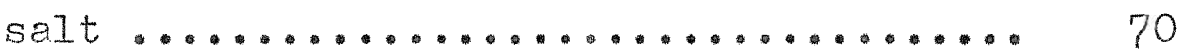

IV Structural parameters of the lameliar phase of lecithin and lecithin-charged linid

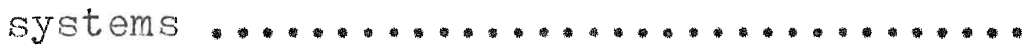

$V$ Variation of the $d$ spacings of the lamellar phase as a function of osmotic pressure

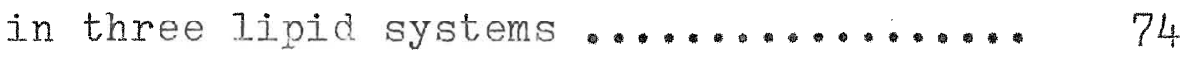

VT Work necessary to bring two charged lipid planes together by distance $d_{w}$ calculated for different regions of force curve.... 78

VII Fistimates of the area per charged group in lecithin-charged Iipid bilayers and evaluation of the varjable .................. 79

VIII Effect of $\mathrm{pH}$ on d spacings of the lamellar phase of $50 \mathrm{~mole} \% \mathrm{PG}$ 


\section{LIST OF ILLUSTRATIONS}

Figure

1 The "fluid-mosaic" model of the membrane

structure $\ldots \ldots \ldots \ldots \ldots \ldots \ldots \ldots \ldots \ldots \ldots \ldots \ldots \ldots \ldots \ldots \ldots$

2. Structure of some representative linids:

phosphatidylcholine (PC), phosphatidylg]ycerol ( $P G$ ), phosphatidylinositol (PI) and

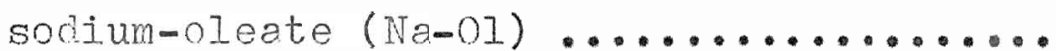

3 Three lamellar phases of phospholipids

$I \beta, I \beta$, and $I \alpha$

4 Interlamellar force versus bilayer sepa-

ration as measured by LeNeveu et al. (1977)

5 A diagram of a lamellar phase of lecithin

with some charged lioids in equilibrium with dextran solution

a) A sketch of $\mathrm{X}$-ray set-up showing geo-

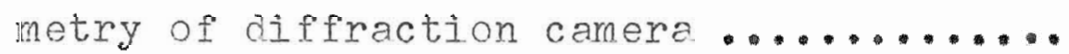

b) A sketch of reflection sphere showing geometry of diffraction

7 A diagram of the specimen holoer showing position of the specimen

8 Structural parameters of the lameliar phase formed by lioid mixtures of various concentrations in water

a) $100 \%$ egg lecithin; 100\% ohosphatidylglycerol, 50 mole\% phosphatidylglycerol

b) 10 and 5 mole\% phosphatidylglycerol, 
Figure

10 mole\% phosphatidylinositol

9 Structural narameters of the lamellar phase

formed by mixtures of lecithin and Na-oleate 36

10 Variation of the d spacing of lanellar phase

in different concentrations of dextran solu-

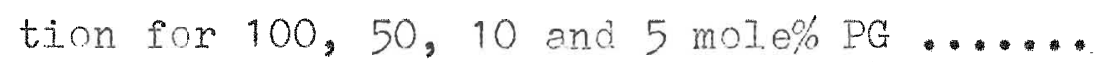

11 Variation of the d spacings of the lamellar

phase in different concentrations of dextran

solutions for 10 mole\% PG, 10 mole\% PT and

$10 \mathrm{~mole} \% \mathrm{Na}$-oleate

12 Calibration curves of dextran solutions

a) Osmotic pressure in cmig versus concentration

b) Logarithm of osmotic pressure versus concentration

13 Relation between the net remusive force $F$

between phosphoti ylglycerol-egg Iecithin

bilayers and their separation $d_{w}$ for 10 and

$5 \mathrm{~mole} \% \mathrm{PG}$

14 Relation between the net repulsive force $\mathrm{E}$

between ljpid bilayers and their separation

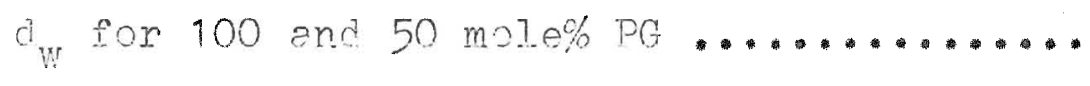

15 Relation between the net repulsive force $F$

between lipid bilayers and their separation

$\partial_{w}$ for $10 \mathrm{~mole} \% \mathrm{PG}, 10 \mathrm{~mole} \% \mathrm{PT}$ and $10 \mathrm{~mole} \%$

Na-oleate 
Figure

16 Variation of the d spacing of lamellar phase in different concentrations of dextran solution for 10 mole\% Na-oleate with salt present in the dextran solution

17 The force curve for 5 mole\% PG showing three regions with different rates of variation of net repulsive force between lipid bilayers and their separarion $c_{w} \ldots \ldots . . . . . . .60$ 


\section{INTRODUCTION}

It has been known for more than fifty years now, that lipids can form bimolecular layers, but it was only in the last decade that a widely accepted model for a membrane structure was proposed. One of the earlier contributions to the understanding of membrane structure was made by Davson and Danielli (1935), who recognized the presence in any membrane of a continuous hydrocarbon phase. Their model was further refined by Robertson (1958) who suggested that the lipid bilayer is coated asymmetrically by different proteins on the inside and outside of the cellular membrane. With the advance of electron microscopic techniques in the 1960 's extensive studies of membrane structure were conducted and it became apparent that Robertson's "unit-membrane" model needed further modification (Stoeckenius and Engelman 1967) In 1972 Singer and Nicolson had enough data to postulate their "fluid-mosaic" model which is considered the most acceptable model to date (Fig.1). In this model, membrane lipids are thought to form a liquid crystalline matrix in which globular proteins are suspended. These membrane proteins can be integral (interacting with hydrocarbon core of the lipid bilayer) or peripheral (interacting with polar head groups). The fluid-mosaic model satisfactorily accounts for observed membrane asymmetry as well as for widely different protein content in various membranes. It also can be 
Fig.1. The"fluid-mosaic" model of the membrane structure postulated by Singer and Nicolson in 1972.

The large irregular shapes represent globular proteins. The small circles represent lipid polar groups and wavy lines the fluid hydrocarbon chains.

Fig.2. Structure of some representative lipids. Phosph atidylcholine ( $P C)$, Phosphatidylglycerol (PG), Phosphatidylinositol (PI) and Sodium-oleate ( $\mathrm{Na}-\mathrm{OI}$ )

Fig.3. Three lamellar phases of phospholipids $I \beta, I \beta^{\prime}$, and $I \propto$. The small circles represent lipid polar groups, the wavy lines the fluid hydrocarbon chains and the straight lines represent the extended and. parallel hydrocarbon chains. 


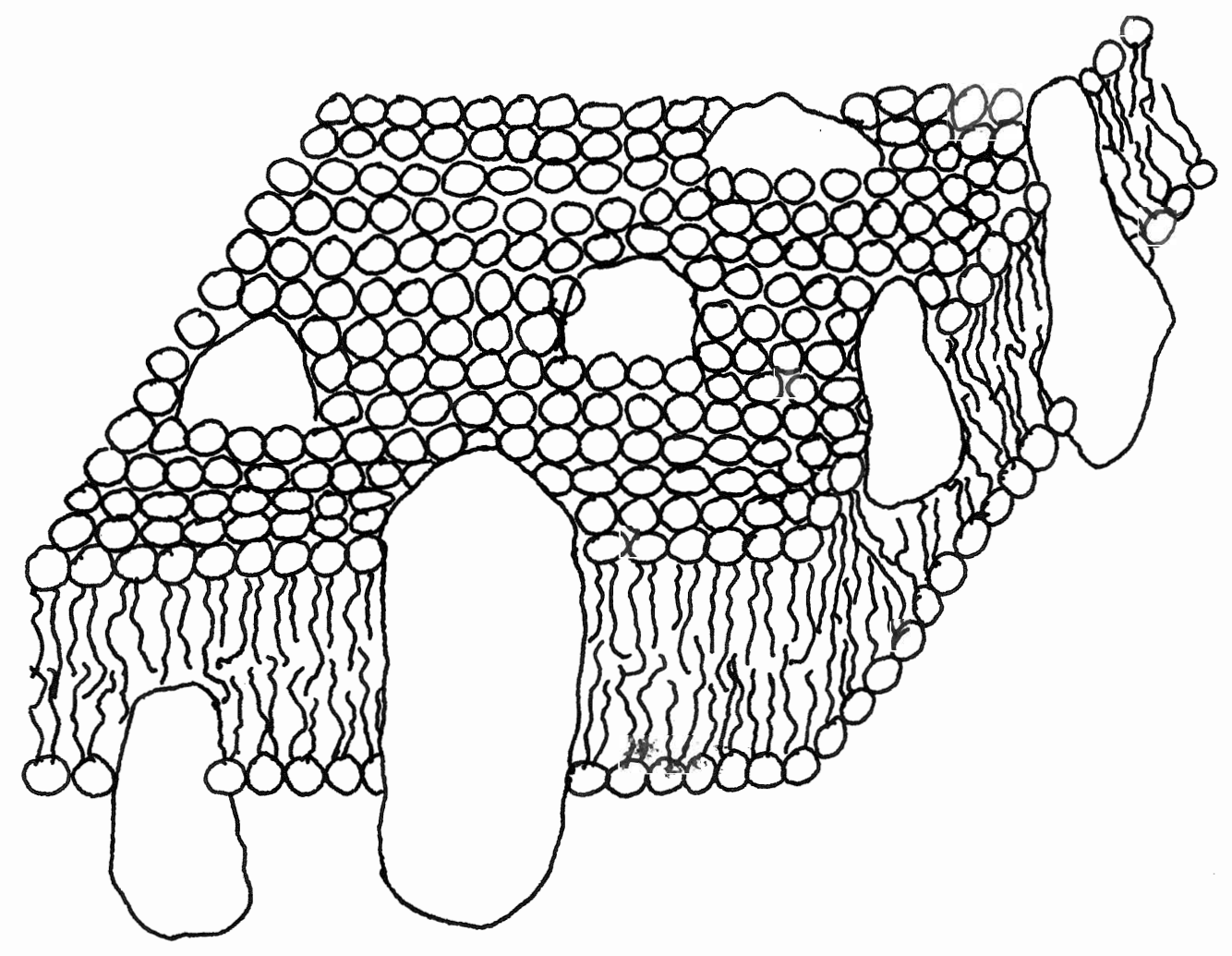

FIG.I. 


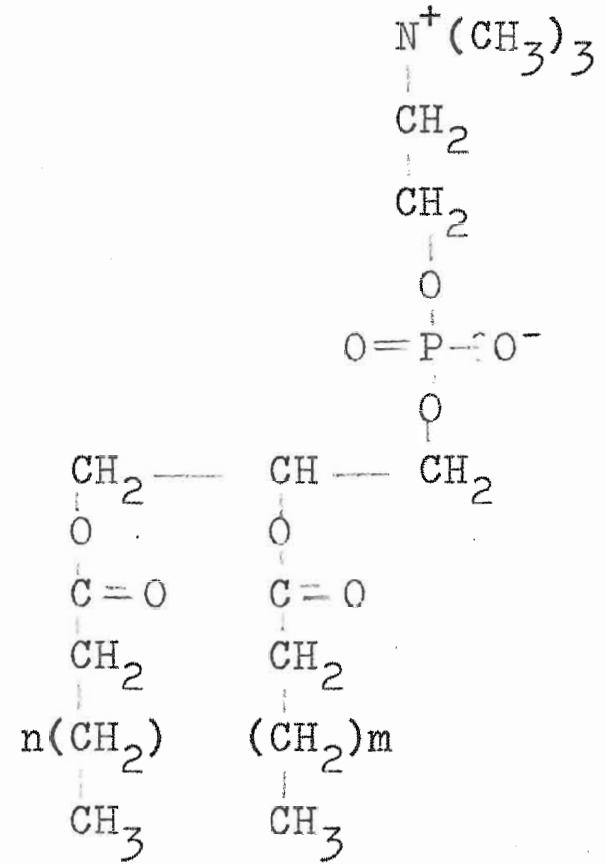

Phosphatidylcholine

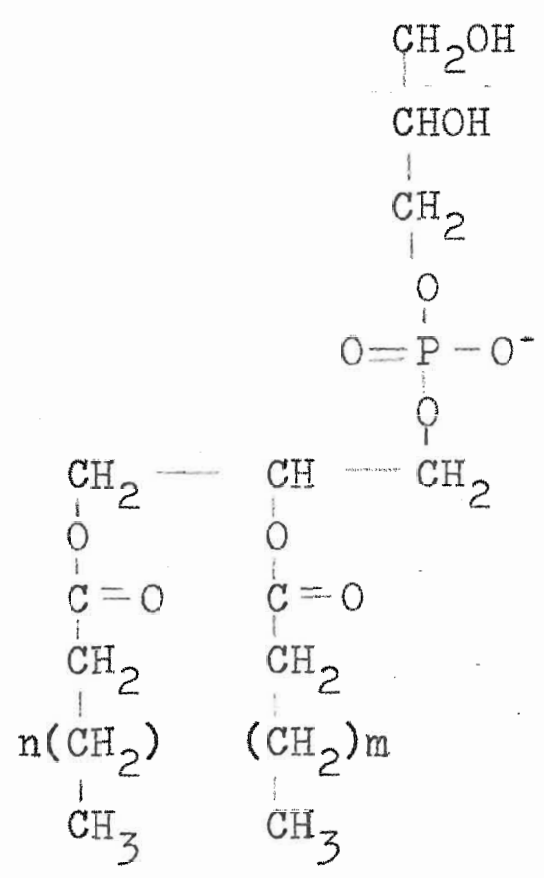

Phosphatidylglycerol
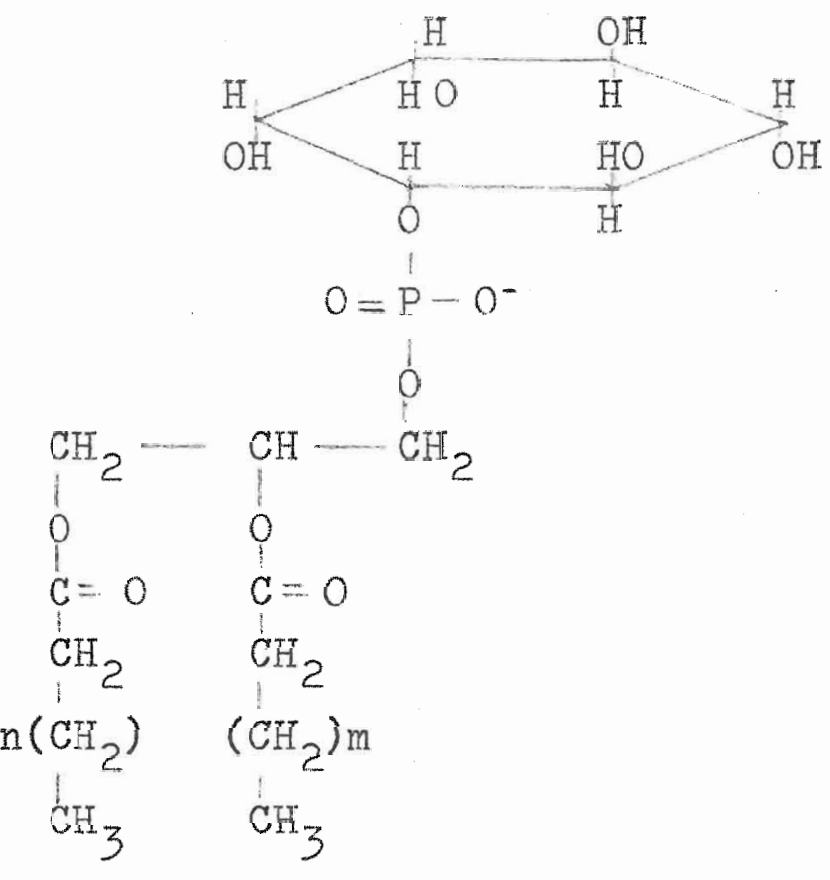

Phosphatidylinositol

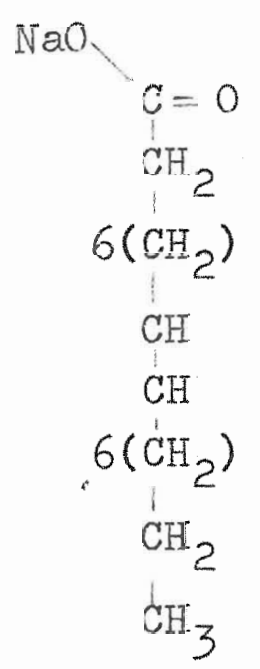

Fig. 2. 


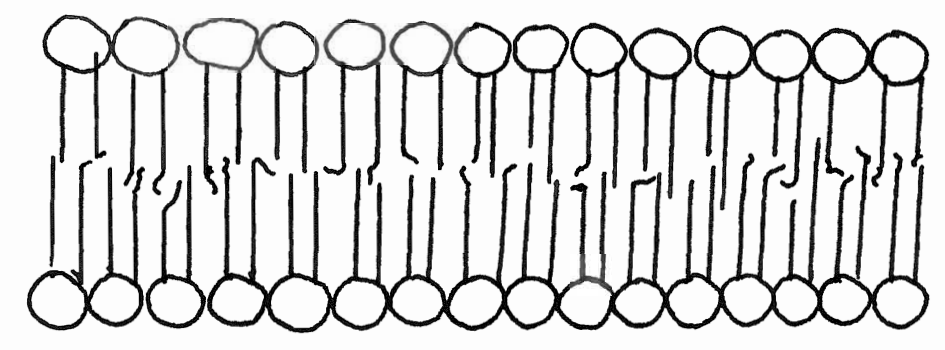

LB PHASE

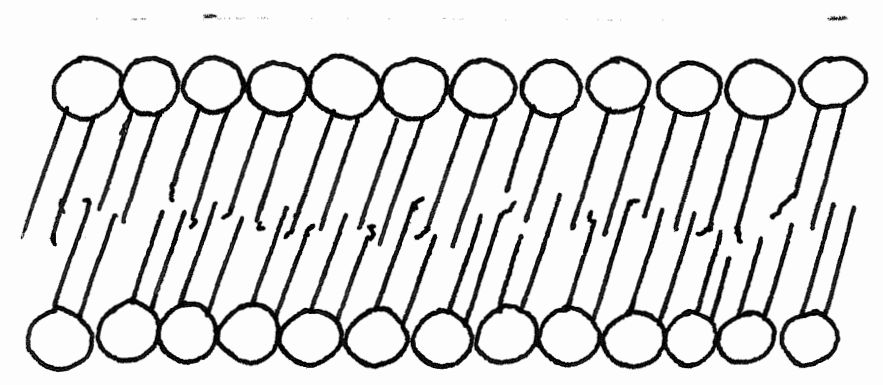

$L B^{\prime}$ PHASE

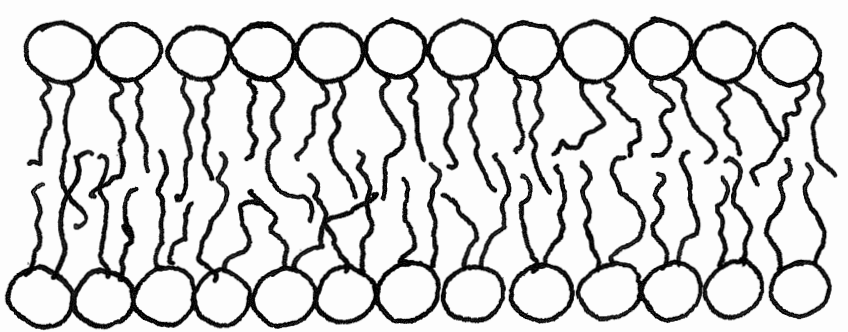

La PHA SE

FIG. 3 . 
used to explain electrical properties of membranes.

It is clear now that all membranes play a very active role in the life of cells. Their structures and functions are many, and therefore an experimental investigation of an intact membrane presents many dificulties. Much easier to interpret are experiments performed on model membranes, built out of one or two lipids and containing no proteins which often complicate the picture. In this thesis all the experiments described were carried out on simple model membranes composed of egg lecithin and selected charged lioids sodium phosphatidylinositol (PI), sodium phosphatidylglycerol (PG) and fatty. acid sodium oleate ( $\mathrm{Na}-\mathrm{Ol}$ ).

Phospholipids are among the most important membrane lipids. They are all similar in that they possess two hydrocarbon chains (10 to 20 carbon atoms long) and an ionizable part referred to as the head group. The differences between these lipids arise from the different structures of thejr head groups and from the length and the degree of saturation of their chains (Fig.2). In water such lipids tend to arrange themselves into lamellar or other three dimentional structures. The first very systematic study of lipidwater systems was carried out by Iuzzati (1968) and his group (Tardieu 1973). They studied biological lipids as well as soaps and detergents. Their experiments, besides establishing the liquid-like conformation of the hydrocarbon chains, also showed that the long range symmetry of the system, in most cases is not affected by the melting of chains when the temperature is increased. 
In natural lipids, the main transition or melting occurs when an ordered gel structure with extended hydrocarbon chains packed parallel to each other and perpendicular to the bilayer plane, changes into a disordered liquid-like structure in which hydrocarbon chains take on a variety of conformations. There is some disagreement as to the amount of order in hydrocarbon chains above the melting point, but there are indications that the upper part of each chain, i.e. the part that is closest to the head group, is more ordered than the free end of the chain (Wilkins et al.1971, Seelig and Seelig 1975, Kohler and Klein 1977).

The different phases through which a lipid system passes on melting are named according to the nomenclature introduced by Luzzati. The gel phase, called $\beta$ phase (Fig.3), is characterized by strong and sharp $\mathrm{X}$-ray Bragg reflections at $S^{-1}=4.2 \AA$ followed by weaker reflections with higher Bragg angles. In the case of the lamellar I $\beta$ phase, this diffraction pattern corresponds to hexagonal packing of extended hydrocarbon chains oriented parallel to the axis perpendicular to the plane of bilayers. It is believed that even in this phase there is a considerable disorder in the region near the center of the bilayer, especially when the lipids forming the bilayer have various chain lengths. Above the melting temperature the hydrocarbon chains are in liquid-like disorder (Tardieu 1973). In this lamellar I $\alpha$ phase, the bilayer structure is preserved and the average orientation of the chains remains perpendicular to the plane of the bilayers. The $I \propto$ phase is characterised by broad band on X-ray photographs at $S^{-1}=4.6 \mathrm{~A}$, which is slightly 
larger than the Bragg lines for the $\beta$ phase, but similar to that observed for liquid paraffins. Levine \& Wilkins (1971) showed that for lipid in the lamellar L $\alpha$ phase the band at $S^{-1}=4.6 \mathrm{~A}$ is anisotropic when the concentration of water is very low, i.e. the intensity of the band varies with the direction of diffraction. In oriented bilayers the maximum intensity in the band appears at $90^{\circ}$ to the direction in which lines arising from the lamellar arrangement are recorded. With increased hydration this anisotropy decreased. This suggests an increased fluidity of the chains with increased hydration of the system.

The thickness of the lipid bilayer of the $L \alpha$ phase is known to be temperature dependent. Terdieu et al. (1973) measured the linear thermal expansion of the bilayer and found it to be negative, $-10^{-3} /{ }^{\circ} \mathrm{C}$. The thinning of the bilayer with increasing temperature was also observed for phases other than lamellar. Tardieu et al. compare this behaviour of lipid bilayers, to the behaviour of a sheet of rubber or polymer subjected to a rising temperature. Such sheets when subjected to heat while they are stretched show thinning of the sheet. This thinning is explained as a result of increased disorder among the fibres or molecules that form the sheet. If the thinning of the lipid bilayer arises in the same manner as the thinning of the sheet of rubber then one has to establish what are the stretching forces that operate on the bilayer.

Another phase reported by Luzzati and investigated by Rand et aI. (1975) and Janjak et al. (1976) is phase I/.'. 
It is similar to the I phase with the hydrocarbon chains extended and in parallel packing, however the orientation of the chains with respect to the plane of the lamellae is not perpendicular as in phase $\mathrm{L} \beta$, but forms a tilt whose angle with the plane of the lamellae increases with increasing water content of the lipid. In X-ray photographs the I $\beta^{\prime}$ phase is characterised, as was phase $L \beta$, by sharp reflection at $S^{-1}=4.2 \AA$, but now the main reflection is followed on the high angle side by a more diffuse band. This phase appears at the pretransition temperature when the system is heated up from the Is $\beta$ phase, and it disappears at the transition temperature when the system goes into the $I \propto$ phase.

More recently a new phase $P \beta^{\prime}$, also called a ripple phase, was reported. Like L $\beta^{\prime}$ phase, the $\mathrm{P} \beta^{\prime}$ is observed omIy with synthetic lipids. The $\mathrm{P} \beta$ 'phase has.. two dimensional symmetry with simple lamellar arrangement of lipids modified by a ripple in the plane of the lamellae (GulikKrzywicki 1975, Janiak et al. 1976). The X-ray pattern for this phase shows a series of weak reflections interleaving the spaces between the strong reflections of the lamellar structure.

The complexity of the membrane structure and the importance of molecular interactions within the membrane between the lipids and between lipids and proteins be- : gan to be understood only at the begining of this decade. McConnell. and his group (McConnell et al. 1972, Scandella 
et al. 1972, McNamee et al. 1973) used spin labels to find the rate of lateral diffusion of phospholipids in both model and natural membranes. They discovered that although lateral diffusion of lipids is fast with a diffusion coefficient $D=1.8 \times 10^{-8} \mathrm{~cm}^{2} / \mathrm{sec}$ the diffusion of lipids from one side of the bilayer to the other is almost non-existent in model membranes and very slow in naturally occuring membranes. Papahadjopuolos and his group (1973, 1974) using differential scanning calorimetry, electron microscopy, spectroscopy and permeability measurements investigated phase transitions in phospholipid vesicles and liposomes made of various lipids. A vesicle is defined as a small, closed, spherical cavity enclosed by a single membrane with a diameter of a few hundred Angstroms. A liposome is a much larger structure of the cross-section of $1000 \AA$ or more. Iiposomes are usually irregular in shape and in most cases they are more stable than vesicles. While vesicle is formed by a single membrane, liposomes may be formed by many layers of membrane arranged uniformly one on top of the other and thus forming a lamellar structure that can be identified in the X-ray photographs. Papahadjopoulos and his group found that the onset of the phase transition in phospholioids subjected to the increasing temperature does not depend only on the composition of the lipids but also on the condition of strain to which the membrane is subjected (there is, for example, much more strain in membrane of small vesicles than in larger liposomes and the transition takes place at a: lower temperature in case of the former than in case of the latter). It was also found that at we transition temperature the 
membranes become rather porous allowing diffusion of ions from one side to the other and easily accommodating foreign molecules, such as peryline, that are used in fluorescence experiments. To explain this effect, Papahadjopoulos suggested, that at the transition temperature the structure of the membrane undergoes a slow change and for some time two lipid phases, liquid and solid, coexist forming a membrane full of defects. The presence of defect structure in membranes at melting point was also recognised by Lee (1977). He argues that at melting temperature both liquid and gel phases are present and each forms separate domains. The mismatched domain boundaries are the sites of defects where exposed hydrocarbon parts of lipids can be found. For this reason fusion is most likely to occur at the melting temperature. Lee points out that the presence of the transition phase with its defect structure accounts for a basic difference between melting processes of crystal.line solids and those observed for membrane lipids. In case of crystalline solids there is a sharp transition between solid and liquid states, while in the case of membranes the transition region is ill defined andtends to stretch over a few degrees.

Another surprising feature of the membrane structure was the presence of asymmetry between the inner and outer faces of some membranes (Casper \& Kirshner 1971, Bretscher 1972). Bretscher found in 1972 that the inner and outer faces of the membrane of red blood cells have different composition. At the same time Michaelson et al. (1972) reported results of their magnetic resonance experiments with 
cosonicated vesicles formed from mixed phosphatidylglycerol and phosphatidylcholine lipids showing that charged phosphatidylglycerol tends to concentrate in the outer surface of the vesicles. Their observations indicated however that there was no segregation of lipids on either side of the bilayer. The more recent study of Iuna et al. (1977) carried out on unsonicated dispersions formed from binary mixtures of charged and uncharged lipids shows, on the other hand, that there is a definite lateral phase separation within the plane of the membrane between charged and uncharged lipids.

The asymmetric distribution of lipids on the two sides of the natural membranes was found also in influenza virus, in bacterial cells and in the bacteriophage PM2 (Grodecki 1973, Rothman 1977). The distribution of lipids in different membranes, however, is not unique as the same Iipids are found on both sides of the membrane bilayer but not in the same amounts. The origin of the observed asymmetry of lipids as well as its stability are not as ye't understood. The results obtained for natural membranes differ from those obtained for model membranes. In model membranes the rate of flip-flop of lipids across the membrane is almost non-exist ent (Rothman 1975) and any asymmetry present cannot be sustained permanentIy. In natural membranes on the other hand, the presence of membrane proteins facilitates diffusion of lipids from one side of the membrane to the other (McNamee et al. 1973). This facilitated flin-flop combined with rapid rates of lateral diffusion can ensure 
stable asymmetry of lipids even if there are only a few sites at which lipids can diffuse across the membrane.

It is, however, not only the interactions that occur within the plane of a single membrane that are important in the biology of membranes. In any complex organism interactions between membranes of neighbouring cells are necessary to allow for specific recognition of cells. Disorder in such interactions may lead to cancerous growth. Stable short-range interactions usually involve specific molecules on the cell surface, though in order to establish such short-range interactions a long-range electrostatic or van der Waals force may play an important role (Parsegian 1973, Weinstein et al. 1977). To understand such long-range interactions model membranes are again of great help as they allow a quantitative study of the energies involved in the interactions. It is only after such quantitative work is done and the forces of attraction and repulsion are identified, that a prediction about aggregation or fusion, adhesion or molecular exchange between cells can be made.

Current studies on vesicle interactions by Papahadjopoulos (1976 a\&b) showed that vesicles made of neutral phosphatidylcholine are more resistant to fusion than vesicles made with charged lipids, and the expected electrostatic repulsion was not found to interfere with the fusion processes. AIso, it was found that the fusion of vesicles or of vesicles and cells was observed only when the vesicles were incubated above the transition temperature of all the lipids involved. The small vesicles interacted more readily than larger multilayer liposomes, probably because the former are under more strain than 
the latter. This means that the fusion is enhanced by destabilisation of bilayer structure. The fusion can also be brought on by the presence of divalent ions such as $\mathrm{Ca}^{2+}$ or $\mathrm{Mg}^{2+}$ or destabilizing agents such as lysolecithin, proteins or dimethylsulfoxide. Both Papahadjopoulos et al(1976a\&b)\& Pagano (1975) made the point that often what was considered to be fusion was just adhesion without fusion in which case some diffusion of lipids between membranes could take place. In some cases molecular exchange by exchange proteins, as reported by Rothman et al. (1975) which causes redistribution of lipids between vesicles, had not been considered and fusion was assumed as the only interpretation of results. Weinstein et al. (1977) argues that true fusion, when positively identified as in his experiments with trapped fluorescent markers, is a rather unlikely event. Even when vesicles were targeted on lymphocytes, with which they were to react, by creating an antibody bridge between haptens carried by vesicles and Iymphocytes, very Iittle fusion was observed. AII the evidence points to some strong forces preventing fusion of stable membranous structures. Only when a bilayer is destabilised, can a fusion take place. It seems therefore, that the one of the requirements for fusion is the presence of an exposed region of hydrocarbon parts at the place of contact of two membranes.

\section{Intermembrane forces.}

The interactions between macroscopic bodies separated by distances much greater than interatomic spaces can be electrostatic or electrodynamic in nature. The theoretical 
treatment of attractive interactions between dielectric materials in a solid or liquid medium was developed by Dzyaloshinski, Lifshitz and Pitaevskii (1959). Their rigorous theoretical analysis has been succsessfully applied by Parsegian $(1973,1975)$ to the problem of the interaction of biological membranes. The presence of attractive longrange van der waals forces is well demonst rated in colloid science by coagulation or floculation of particles. It is known however that beside the attractive forces there exist some strong repulsive forces at close approach, which create an energy barrier keeping coagulated particles separated. Barclay et al. $(1970,1972)$ indicated that this energy barrier arises due to strong hydration forces.

One of the first quantitative measurements designed to find the values of such forces was carried out by Tabor and co-workers. They measured the long-range forces between two crossed cylindrical sheets of molecularly smooth muscovite mica in vacuo, in air and in aqueous solutions (Tabor et al.1969, Israelachvili et al. 1972 \& 1976). Their experiments showed that attractive van der Waals forces were of the order of magnitude predicted by the theory. They also observed a strong repulsive force in the region of the Debye length (the Debye length is the distance over which the screened potential decreases by the factor e, where e=2.71828). The observed repulsive force decayed exponentially outside the Debye length $\lambda(\lambda \cong 10 A)$ in accordance with predictions of the double layer theory. Recent convincing experimental evidence disclosing 
the character and magnitude of such long-range forces was presented by LeNeveu et al. (1976 \& 1977). In their experiments the interacting surfaces were lipid bilayers of model membranes. It was recognised that the stable lamellar multilayers formed by neutral phosphotidylcholine in water, can exist only if there is a balance between lorig-range attractive van der Waals forces and some unknown repulsive force. To change the bilayer separation from its equilibrium position in water of $27.5 \AA$, an osmotic pressure $\pi$ was applied to the lamellae by means of dextran in extralamellar water phase. The separations between bilayers were measured by $\mathrm{X}$-ray diffraction methods. Since the external osmotic pressure was forcing bilayers together it was acting in the same direction as the van der Waals attractive force $F_{A}$ and in the opposite direction to a repulsive force $F_{R}$. A simple equation gives the following balance of forces:

$$
\left|F_{R}\right|=\left|F_{A}\right|+|\pi|
$$

Now $\pi$ is a known measurable force while both $F_{A}$ and $F_{R}$ are unknown. From calculations using Dzyaloshinski, Lifshitz and Pitaevskii's theory of van der Waals forces and from observations made by LeNeveu et al. the inequality $\pi \gg F_{A}$ was established for separations (a few Angstroms or more) smaller than equilibrium separation, in the absence of dextran, of $27.5 \AA$ for the lecithin-water system. This verified the approximation $\pi \cong \mathrm{F}_{\mathrm{R}}$ for those small separations. Because $F_{R}$ was found to decrease exponentially, extrapolation of this force to the equilibrium separation 
where $\pi=0$ gave the value of $F_{A}$, since at that point $F_{R}=F_{A}$. The plot of $\log _{10} \pi$ vs. $d_{W}$ gave the equation:

$$
F_{R}=P_{0} \exp \left(-d_{W} / 1.93\right)
$$

where $P_{0}=1.0 \times 10^{11}$ dynes $/ \mathrm{cm}^{2}$ and $a_{w}$ is given in Angstroms. The attractive $\mathrm{F}_{\mathrm{A}}$ force was assumed to be of the form

$$
F_{\mathrm{A}}=-A_{\mathrm{H}} / 6 \pi \mathrm{d}^{3}
$$

where $A_{F}$, the Hamaker coefficient, was evaluated to be of the order of $6.5 \times 10^{4} \mathrm{dynes} / \mathrm{cm}^{2}$, (Fig.4).

The experiments by LeNeveu et al. (1977) introduced a new technique for study of forces between phospholipid bilayers. The object of the present thesis was to study quantitatively the electrostatic interactions between charged lipid bilayers using this new technique.

The presence of electrostatic forces between bilayers of brain lipids was demonstrated by Palmer \& Smith (1941) who found that those lipids swelled indefinitely in distilled water but shrank when exposed to solutions of progressively higher ionic strength. Then in 1969 GulikKrzywicki showed that even small amounts of charged phospholipids added to egg lecithin bilayers provide sufficient electrostatic repulsion to cause the swelling of bilayers to large separations.

Recently the interactions of red blood cells with a solid surface of varying electrostatic potential were studied by Gingell \& Fornes (1976). Also some indirect measurements of the surface potential of cells and model membranes were made by Castle et al. (1976) and McLaughlin (1977). 
Fig.4. Interlamellar force versus bilayer separation as measured by LeNeveu et al. Nvumber of water nolecules per lecithin molecule for a given bilayer separation $d_{w}$, is given by the top scale. ( LeNeveu, Rand,Parsegian and Gingell, Biophys.J. $(1977), 18,209-230$.

Fig.5. A diagram of a lamellar phase of lecithin with some charged lipid, in equilibrium with dextran solution. The forces acting between lamellae are indicated, $F_{A}$ represents the attractive van der Waal's force, $F_{F}$ the repulsive electrostatic force, $F_{H}$ the repulsive hydration force and $F_{\pi}$ the osmotic force of dextran pushing bilayers together.

The open circles represent lecithin head groups and the black small circles represent charged lipid head groups; the wavy lines represent the fluid hydrocarbon chains. 


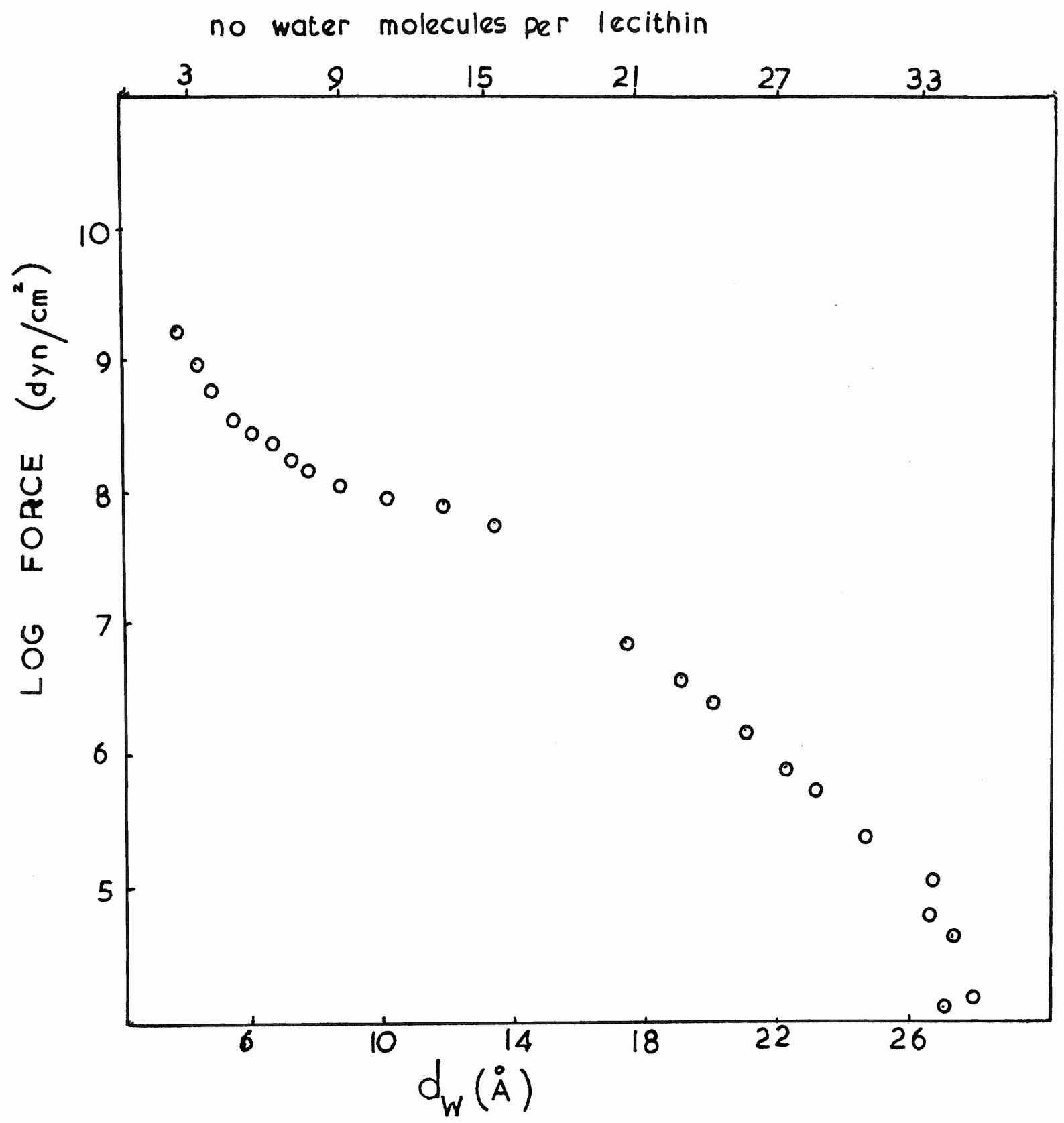

$F \mid G .4$. 


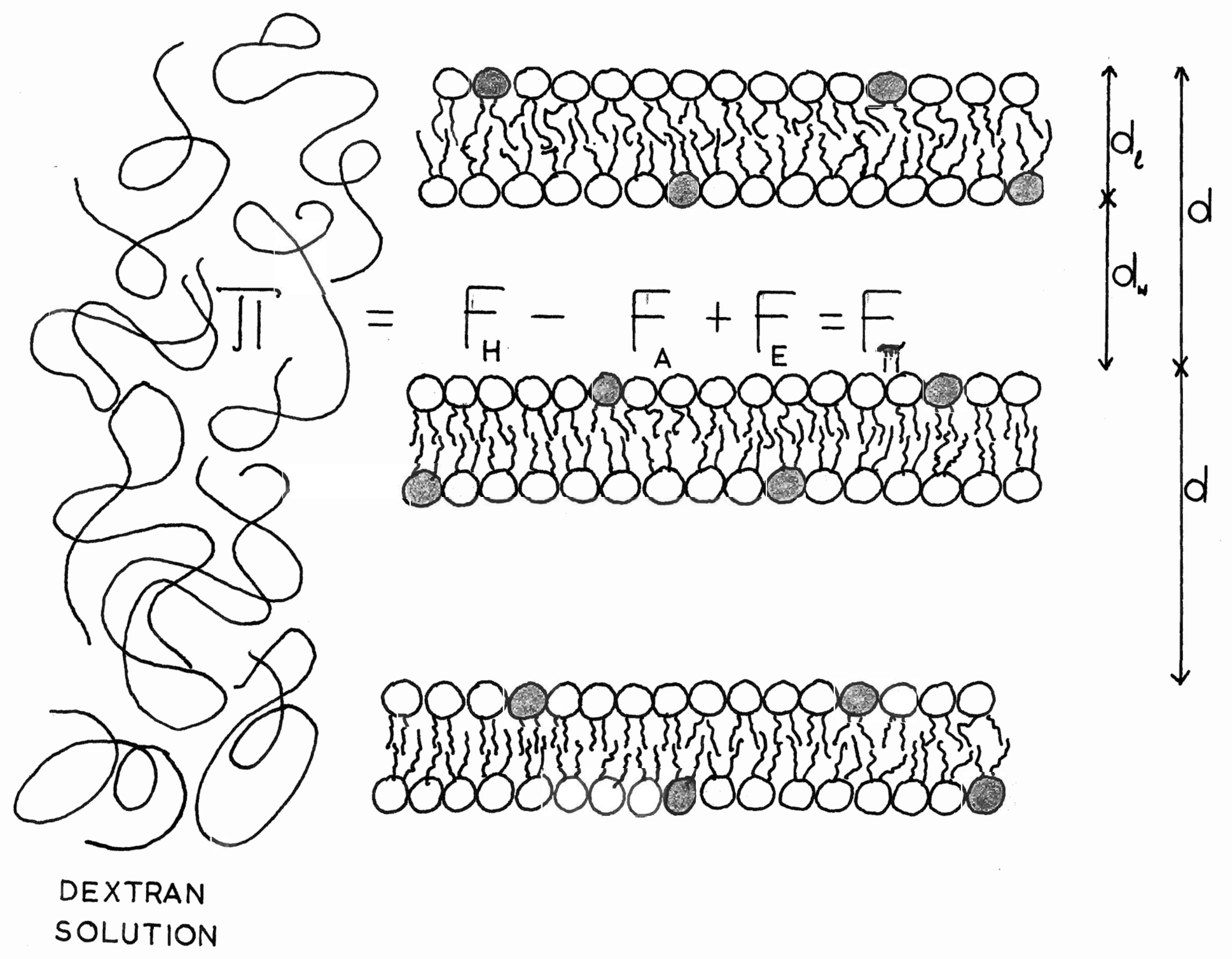

FIG. 5 . 
Theoretical calculations of electrostatic forces beteen charged planar layers were carried out by Ninham et al. (1971), Goulden (1976) and Boguslawski et al. (1976)。 The work of McLaughlin et al. (1976) showed convincingly that the theory of the diffuse double layer accounts remarkably well for the effects of the alkaline earth cations on the surface potential of the charged artificial membranes.

The experiments presented in this thesis give further evidence that the simple double-layer theory explains the physical observations quite well. Our ability to measure the magnitude of the electrostatic forces for the first time gave a new understanding of the involvement of different types of forces in the intracellular interactions. The balance of force equation used here is a modified equation used by LeNeveu et al. (1977)

$$
\left|F_{E}\right|+\left|F_{H}\right|=|\pi|+\left|F_{A}\right| \text {. }
$$

A new term $F_{\mathrm{E}}$ accounts for electrostatic repulsion between bilayers caused by the presence of charged lipids and the other forces are as described above, (Fig.5). 


\section{MATERTAISS AND METHODS.}

1.1 Mixing of charged lipids with egg lecithin.

The egg lecithin used in these experiments was prepared by the method of singleton et al. (1965). Fresh lecithin was prepared every few months. The lecithin and all the lipids used were stored dry under a nitrogen atmosphere at $-20^{\circ} \mathrm{C}$. The purity of the lipids was monitored by thin layer chromatography. The chromatography plates were prepared by coating glass plates 0.5 mm thick with silica gell and then baking them in an oven. The solvent used was a mixture of chloroform ( $97.5 \mathrm{~mL})$, methenol $(37.5 \mathrm{~mL})$ and water ( $8 \mathrm{mI}$ ) with a drop of HCl.

The charged lipids used in our experiments were: sodium phosphatidylinositol (PI) extracted from pig erythrocytes, a generous gift of Dr. D.o. Tinker of the University of Toronto; sodium phosphatidylglycerol (PG) prepared from egg lecithin, a generous gift of Dr. D. Papahadjopoulos of Roswell Park Memorial Institute, Buffalo; and synthetic fatty acid sodium oleate (Na-OI) obtained from Serdary (London, ont.).

To prepare the lipid mixture, dry lecithin was dissolved separately in chloroform and the charged lipid was dissolved separately in chloroform with two drops of methanol. The two solutions were mixed together and then dried on a rotary evanorator until all solvent was removed and dry film formed on the sides of the flask. 
Further drying of the lipid took place by leaving the sample overnight in vacuum. The mole percent of charged lipid in neutral lecithin was evaluated from the equation:

$$
\text { Mol\% charged lipid }=\frac{\text { Moles charged lipid }}{\text { Moles charged lipid + Moles lecithin }}
$$

Molecular weights used were: lecithin - 790, PI - 865, $P G-777$ and $\mathrm{Na}-01-304$.

The mixtures of lecithin with sodium oleate were thought to be less reliable than those prepared with the two charged phospholipids (PI \& PG) because of known tendency of sodium oleate to separate into micelles in lipid-water mixtures. We had no evidence that such separation did. occur, but if some sodium oleate separated into micelles then the charge density in the lecithin bilayers would be uncertain.

1.2 Preparation of dextran solutions.

Dextran solutions were prepared using dextran T2000 from Pharmacia Fine Chemicals, Sweden (M.W. 2,000,000). Spectral analysis of this powder showed that the calcium level was below $1 \mathrm{ppm}$. Even if 1 ppm of calcium was present, then in the case of $15 \%$ dextran solution and lipid containing 10 mole\% of charged head groups ( $3 \mathrm{gm}$ of solution were in contact with $10 \mathrm{mg}$ of lipid), only one $\mathrm{Ca}^{2+}$ ion would be available for 1000 charged heads.

The water used to prepare dextran solutions was deionized and glass distilled. The presence of any ionic species in the water was checked by measuring conductance (Tabje I). 
To lower the conductance of the dextran solutions, they were treated with mixed bed deionizer resins. In order to keep the variations in the dextran concentrations of our solutions to a minimum, they were prepared by dilution from ə. stock of high cextran concentration of about $40 \%$. The final concentrations of the solutions were checked with an Abbe refractometer. The refractive indices are lister in Table II.

In a few experiments the effect of electrolytes on the swelling of lipids was checked. For these experiments some salts were added to dextran solutions. The following solutions were used: $0.1 \mathrm{M}$ and $0.01 \mathrm{M}$ in $\mathrm{NaCl}, 2.0 .1 \mathrm{M} \mathrm{Na} \mathrm{NPO}_{4}$ buffer solution and $75 \mu \mathrm{M}$ and $750 \mu \mathrm{M} \mathrm{CaCl}$. The results are shown in Table III and Figs 12 \& 16.

\section{$1.3 \quad x-r a y$ diffraction.}

All the $\mathrm{X}$-ray photographs were recorded with copper $K \alpha$, radiation, $\lambda=1.541 \AA$. A standard Guinier camera was used. The highest Bragg angle measured was $9.6^{\circ}$, corresponding to the band at $4.6 \AA$ arising from the packing of hydrocarbon chajns. The diagram of Fig.6a shows the experimental set up. A curved crystal monochromator was used to select the wavelength and to focus the $\mathrm{X}$-ray beam on the sample. The collimation was regulated with two slits.

Two cemeras were used, one with radius $\mathrm{R}=6.25 \mathrm{~cm}$ and the second with radius $\mathrm{R}=3.125 \mathrm{~cm}$. Medical $\mathrm{X}-\mathrm{ray}$ film by Kodak was used and developed according to standard procedures. Long exposures of several hours and often over-night were needed and to eliminate background scatter- 
Fig.6. a) A sketch of $X$-ray set up showing geometry of the diffraction camera.

b) A sketch of reflection sphere showing geometry of diffraction. $R$ is the radius of the camera and $i$ is the distance measured along the film.

Fig.7. A diagram of a specimen holder showing position of the specimen. Also shown is the arrangement used for equilibration of lipid sample with dextran solution inside the specimen holder. 


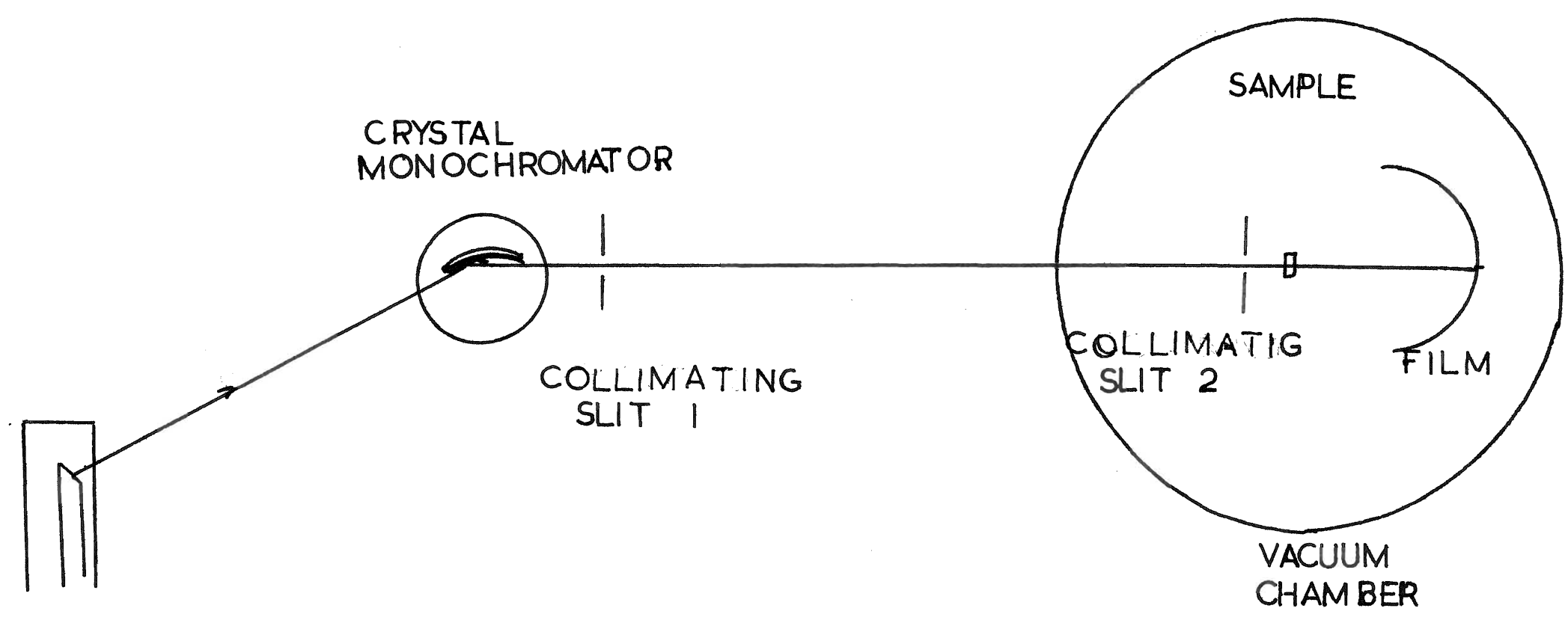

$X$ RAY

GENERATOR

FIG. 60 . 


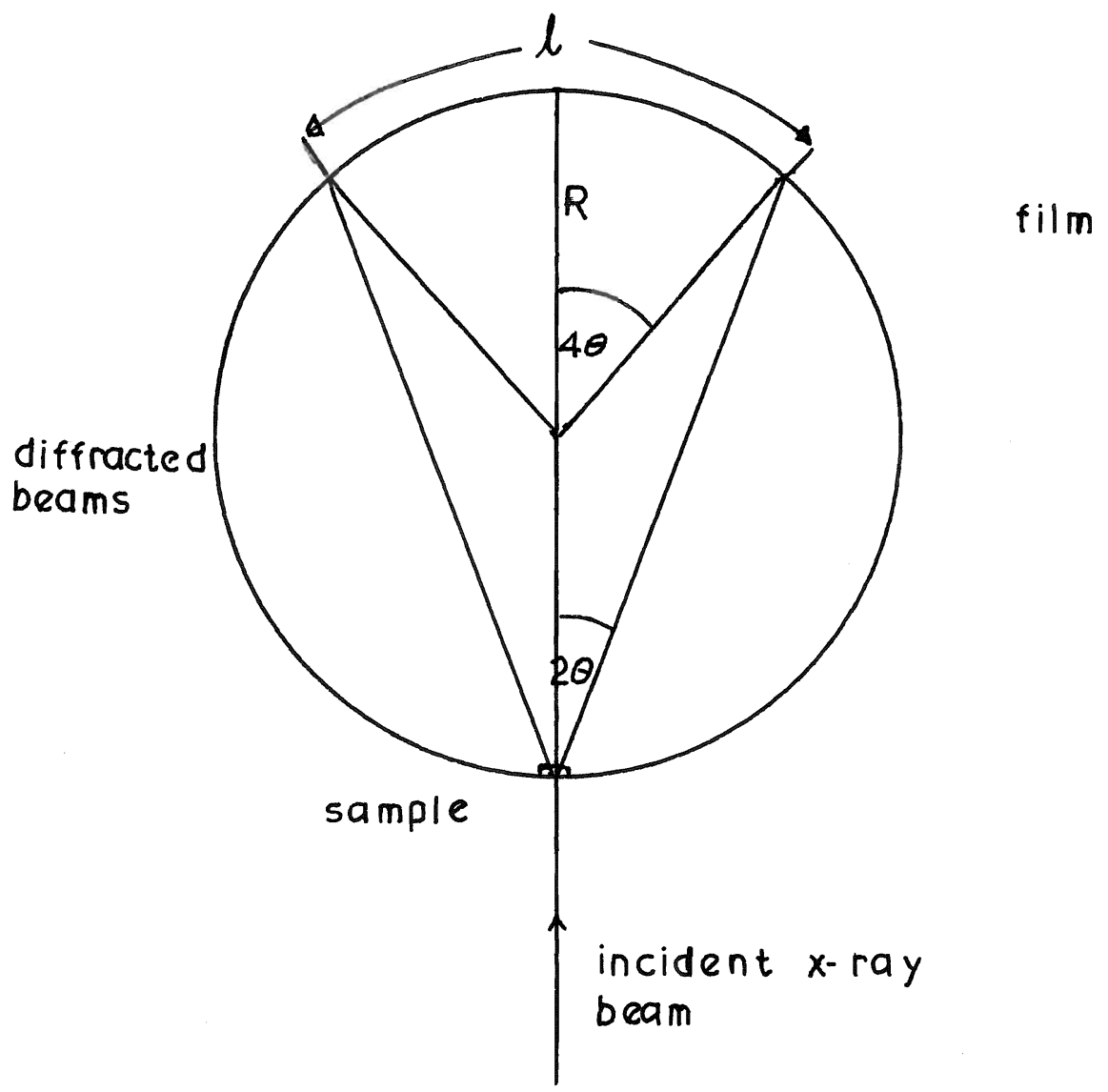

FIG. 6 b. 


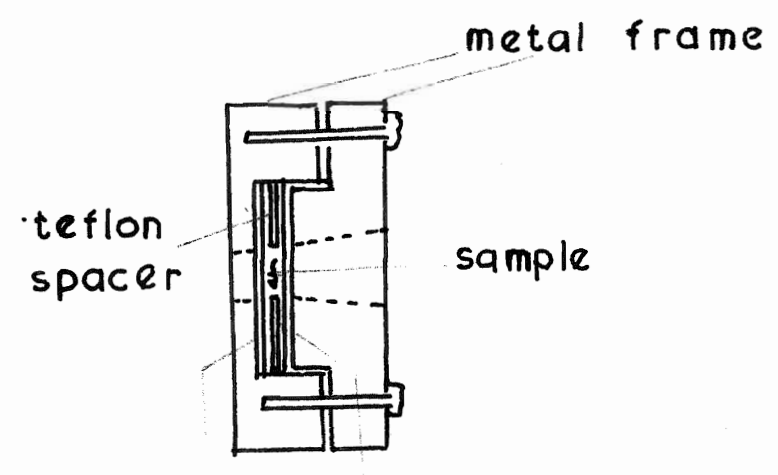

mica windows

SIDE VIEW

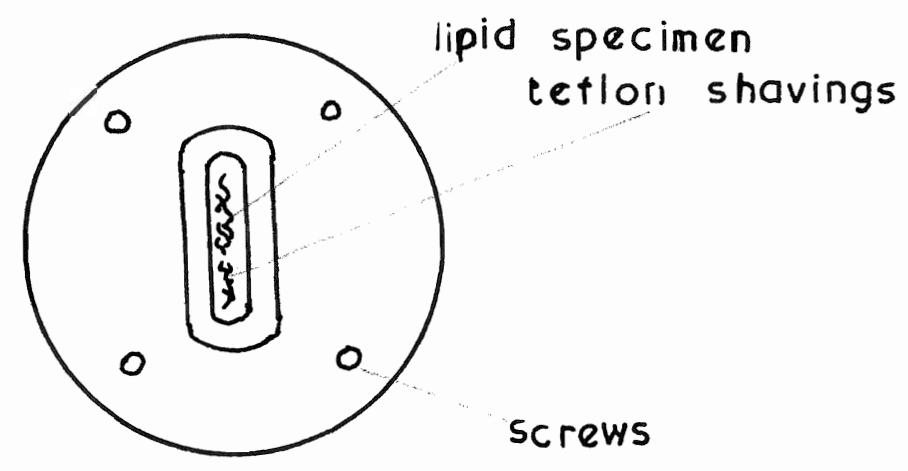

TOP VIEW

dextran solution

membrane

paraffin film

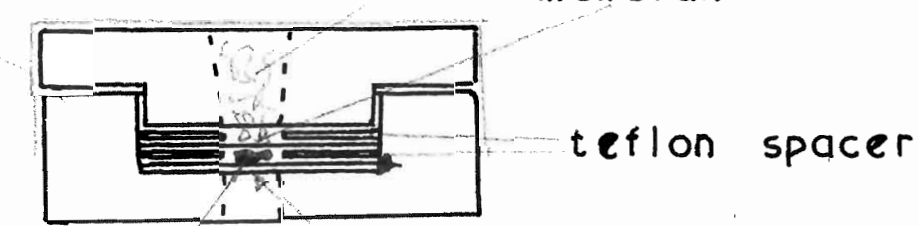

sample mica window

$F \mid G .7$. 
ing, the sample and the fjlm were housed in an evacuated chamber. The sample to film distance is twice the radius of the camera as shown in Fig. 6 .

Since $n \lambda=2 d \sin \theta$

$$
1 / \lambda=2 \sin \theta / \lambda \cong 2 \theta / \lambda \text { for } \operatorname{small} \theta
$$

Now $\theta$ is related to the distance on the film between two measured lines $\boldsymbol{l}$, and we can write:

$$
\theta=\ell / 8 \mathrm{R}
$$

For $R=6.25 \mathrm{~cm} \quad 1 / d=0.002597 \ell$

for $R=3.125 \mathrm{~cm} \quad 1 / d=0.005194 \ell$

where $\ell$ is in $\mathrm{mm}$ and $d$ in Angstroms.

The film to sample distance was not always constant as often more than one film was loaded into the camera. Also the separations between the lines on the film could be affected by the shrinkage of the film in the developing process. To avoid such errors that would affect the measured a spacings, some dry teflon powder was included in the sample holder to give a standard teflon line. The teflon spacing giving this line was calculated from a berylium acetate diffraction pattern as given in Appendix II.

The lamellar phase of a lipid appears in $\mathrm{X}$-ray diffraction pattern as a series of lines corresponding to the crystallocraphic repeat distance $d$, where $d=d_{l}+d_{w}$ * Such a lamellar phase is composed of lipid bilayers of thickness $d_{l}$ and interleaving water layers of thickness $d_{w}$ as shown in Fig. 5. In their original analysis of the lamellar lipid pattern Rand and Juzzati (1968) defined de as the thickness of the lipid layer that the lipid mole- 
cules woul occupy in the repeat period without water.

Thus $d_{l}=\phi d$,

where $\phi$ is the volume percent of lipid. If the composition of the water-lipid mixture is known from careful weighing of dry lecithin and water, and the partial specific valumes of lecithin and water are also known one can write:

$$
\phi=\mathrm{v}_{\mathrm{PC}} \mathrm{W}_{\mathrm{PC}} /\left(\mathrm{v}_{\mathrm{PC}}+\mathrm{v}_{\left.\mathrm{H}_{2} \mathrm{O}_{\mathrm{H}_{2} \mathrm{O}}\right)}\right.
$$

where $w_{P C}$ and $w_{H_{2}} \mathrm{O}$ are weights of lecithin and water respectively and $\mathrm{v}_{\mathrm{PC}}$ and $\mathrm{v}_{\mathrm{H}_{2} \mathrm{O}}$ are their specific volumes. If the partial specific volume of lecithin is assumed to be equal to that of water than expression for $\phi$ reduces to:

$$
\phi \cong \mathrm{w}_{\mathrm{PC}} /\left(\mathrm{w}_{\mathrm{PC}}+\mathrm{w}_{\mathrm{H}_{2} \mathrm{O}}\right)=\phi^{\prime}
$$

The value of $\phi$ (calculated using $v_{P C}=0.987$, Tardieu 1973) differs from $\phi^{\prime}$ as follows:

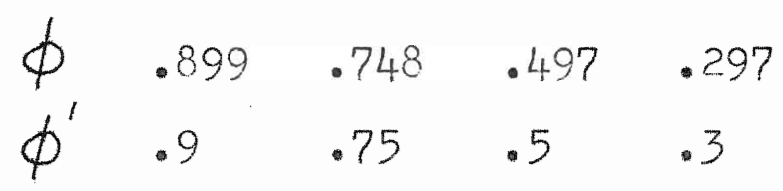

which, in most cases, gives error smaller than $1 \%$.

1.4 Preparation of X-ray samples.

The lipid-water samples, in equilibrium under the osmotic pressure of dextran were prepared in two ways. In one method the lipid was placed in direct contact with dextran solution of known concentration (about 20mg of lipid to $200 \mathrm{mg}$ of dextran) in small weighing bottles with tightly fitting caps. The system was allowed to equilibrate for a few days and the concentration of dextran so- 
Iution was checked by refractometry. The osmotic pressure was calculated from this final equilibrium concentration. After equilibration the lipid along with some of the dextran solution was transfered to the specimen holder (Fig.7) where the sample was mounted between two mica windows separated by a flat ring of teflon. All the touching surfaces were sealed with vacuum grease. Two outside metal discs screwed together kept the sample unchanged for weeks. In the second method the dry lipid was mounted directly in the sample holder and remained separated from the dextran solution by a dialysis membrane as shown in Fig.?. After 48 hours or more the dextran solution was removed, its concentration checked by refractometry, and if necessary a new solution used to replace the old one. Finally when the concentration of the dextran solution did not show change, most of the dextran solution was removed and the second mica window installed on the membrane side of the sample. The whole specimen holder was then screwed together and secured with vacuum grease.

It was observed that for high dextran concentrations swollen lipids hold together well and were easy to transfer from weighing bottles to specimen holders. For lower dextran concentrations ( $18 \%$ or less) however, the lipid tended to disperse in the dextran solution and lots of dextran was transfered along with the lipid into a sample holder. This resulted in weak diffraction Jines and extra background scattering. Also the exact dextran concentration, as measured with the Abbe refractometer, was difficult to 
establish because the linid present in the dextran interfered with the refraction pattern. There were indications that for these lerge swellings of lipids, dextran molecules penetrated into the interlamellar water space. When tester, the slightly swollen lipids, after drying gave the expected dry lipid spacings, but those highly swollen, dispersed lipids, when dried gave rather irregular and diffuse $\mathrm{K}-\mathrm{ray}$ patterns. Therefore for dilute dextran solutions the second method of preparing samples was preferable.

To check swelling against very high osmotic pressures, higher than those realized with dextran solutions (i.e. $\sim 10^{7}$ dynes $/ \mathrm{cm}^{2}$ ) a pressure cell, develloped in this laboratory, was used (Fuller et al. 1978). In the cell the dry lipid was squeezed by stainless steel piston against a dialysis membrane supported by a stainless steel grid. Bulk water was available on the other side of the membrane. Any flow of water into the lipid was against a constant pressure applied mechanically to the lipid in the pressure cell. After equilibration, the sample was transfered to the $\mathrm{X}$-ray sample holder and the swelling of the lipid recorded. The pressuresused with this pressure cell were $1 \times 10^{8}$ and $1.2 \times 10^{8}$ dynes $/ \mathrm{cm}^{2}$ (Table V). 
2. RESULTS AND INTFRPRETATION.

In all the lipid systems studied only the lamellar phase with melted hydrocarbon chains was observed. In general only a single lamellar phase was present, but in some cases more than one phase was observed as discussed in the following sections.

2.1 The lipid-water phase diagrams.

The neutral lipid used in all systems studied here was egg lecithin and Fig.8a gives the phase diagram for this lipid. The measured d spacings are plotted together with the calculated values of lipid bilayer thickness $\mathrm{d}_{\boldsymbol{l}}$, and of the water filled distance separating bilayers, $d_{w} \cdot$ The maximum spacing of about $63 \AA$ is reached at the concentration of lecithin in water of $55 \%$ as previously shown (LeNeveu et al. 1977). Any additional. water does not affect the d spacing and must stay out of the lamellar structure and in a separate water phase.

Fig.8 shows phase diagrams for lecithin containing $P G$ and $P I$, all the curves have the same shape. When bilayer thicknesses are calculated the minimum $d_{l}$ value of $36 \AA$ is found to be the same for low concentrations of $P G$ and PI in lecithin as that for pure lecithin. However for 50 mole\% $P G$ in lecithin and for pure $P G$ the lipid bilayer thins only to $38 \AA$. This was a surprising feature since both lecithin and $P G$ were assumed to have the same hydrocarbon chain distribution and the same specific v.lumes. A thicker Jipid bilayer means tighter packing of 
Fig.8. Structural parameters of the lamellar phase. formed by lipid mixtures of various concentrations in water. d - lamellar repeat distance; $a_{\ell}$ - bilayer thickness; $\mathbf{S}$ - area available to exch lipid molecule at the bilayer-water interface calculated from a formula given by Luzzati et al. (1968):

$S=\left(M \cdot W \cdot \mathrm{x} \mathrm{v}_{\mathrm{sp}} \mathrm{X} 10^{24}\right) /($ Avogadro No. $\mathrm{x} \mathrm{d} / 2)$ Molecular weights (M. W.) were assumed to be 790 and 777 for $P C$ and $P G$ respectively and $v$ sp (the specific volume) was taken to be $1 \mathrm{~cm}^{3} / \mathrm{gm}$ for both.

a) $100 \%$ egg lecithin $x$; 100\% phosphatidylglycerol $(\mathrm{PG}) \Delta ; 50$ mole\% $\mathrm{PG}$;

b) $10 \mathrm{~mole} \% \mathrm{PG} \bullet ; \mathrm{mole} \% \mathrm{PG}$; $10 \mathrm{~mole} \%$ phosphatidylinositol (PI) 0 .

Fig.9. Structural parameters of the lamellar phase formed by mixtures of lecithin and Na-Oleate ( Na-OI)

10 mole\% Na-OI $x$; 5 mole\% Na-Ol

5 mole\% Na-OI O (as measured by N.Fuller);

$6 \mathrm{~mole} \% \mathrm{Na}-01 \Delta$.

d, $d_{\ell}$ and $s$ are defined as in Fig. 8.

Marked are also two points when instead of pure water a solution of calcium chloride was used with 10 mole\% $\mathrm{Na-Oleate.}$ 

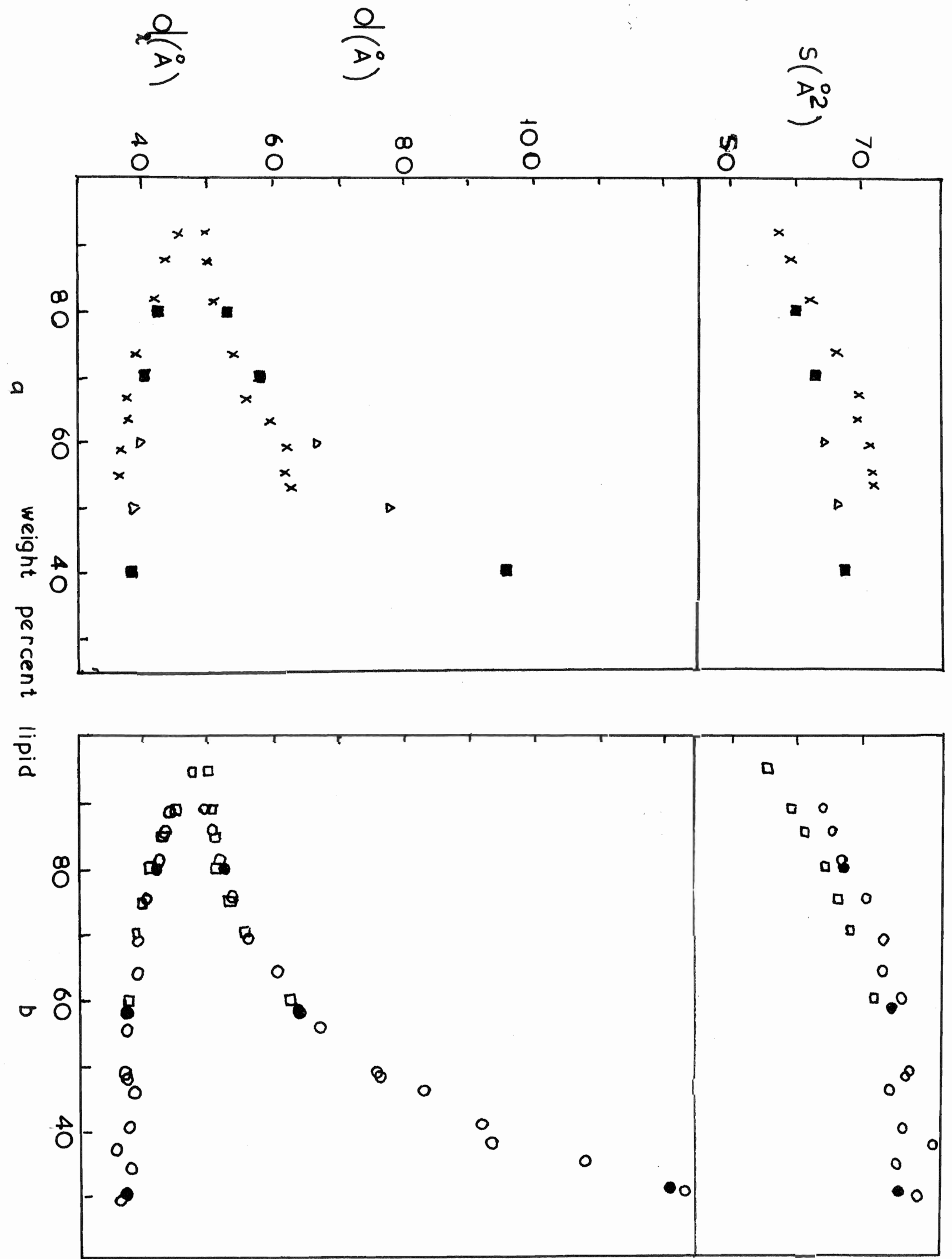

$F \mid G .8$. 


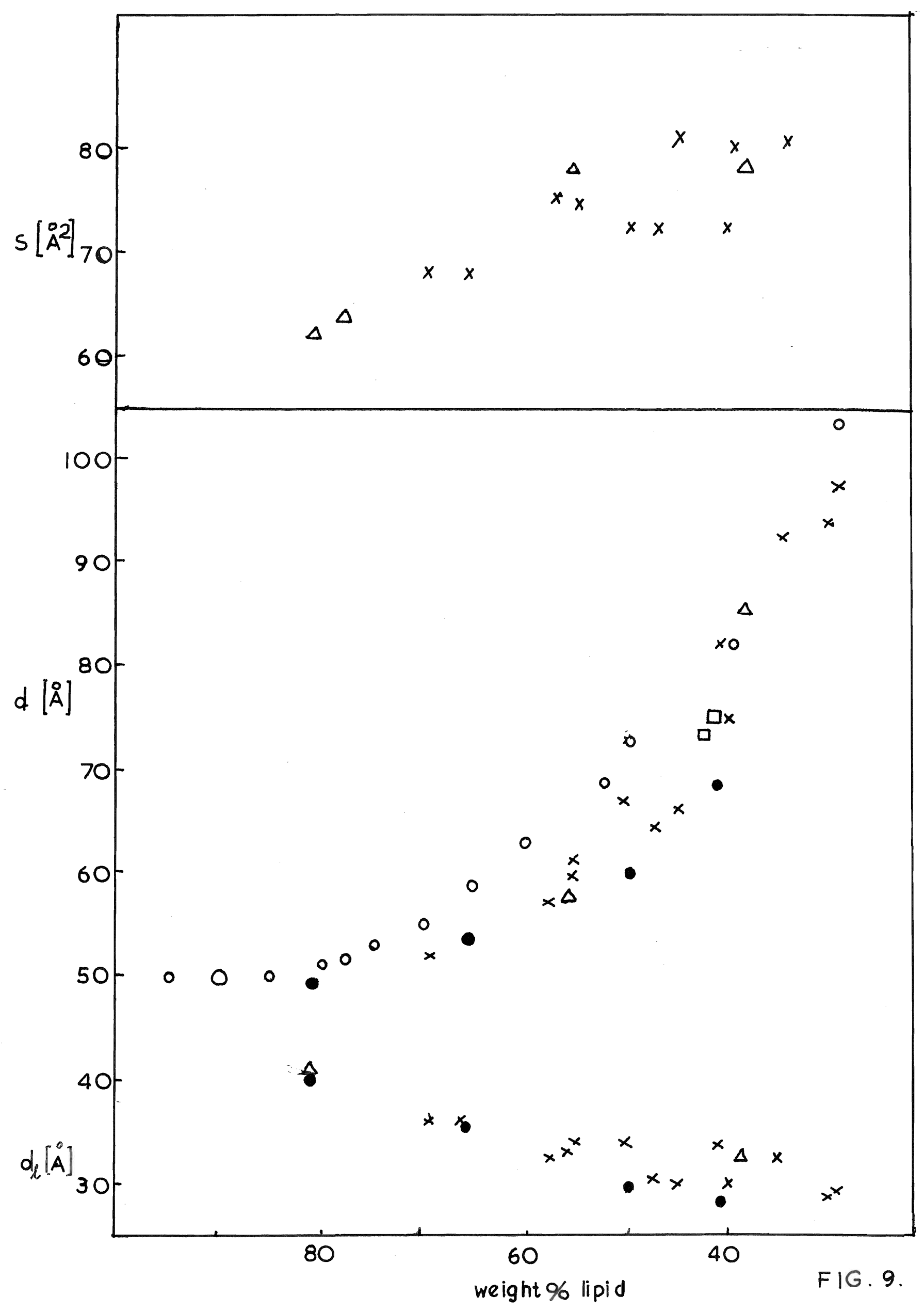


the head groups. The calculated areas per head group, S, are plotted in Fig.8a. For PC the maximum $S$ value is. $72.5 \AA^{2}$ while for $P G$ it is only $67.5 \AA^{2}$. Only the increase of specific volume of $P G$ by $8 \%$ would make the two areas equal, and such a difference between the two specific voIumes is not expected.

Fig.9 gives the phase diagrams for the lecithin-Naoleate system. Although Na-oleate molecules must be incorporated into lecithin bilayers as shown by the swelling of the lipid, there were some indications of randomness in the amount of Na-oleate incorporated. There was always a worry that some Na-oleate might have been lost in micelles instead of going into bilayers, since da-oleate has high solubility and low critical micelle concentration in water. Pig.9 gives also the bilayer separation $d_{w}$ and the bilayer thickness $d_{l}$ calculated from the lattice spacing d for each lipid water concentration as described in methods. 2.2 Swelling of lipid against osmotic oressure of dextran.

Figs 10 and 11 give the d spacings for different lipid systems plotted against the corresponding concentrations of the dextran solutions with which they were equilibrated. As described in section 1.4 the samples were orepared in three ways:

(i) lipid was in direct contact with dextran solution while equilibrating in weighing bottle,

(ii) lipjd was separated from dextran solution by a dialysis membrane and during the equilibration process in the $\mathrm{X}$-ray sample holder only 
Fig.10. Variation of d spacing of the lamellar phase in different concentrations of dextran solution for $100 \mathrm{~mole} \% \Delta ; 50 \mathrm{~mole} \%$; $10 \mathrm{~mole} \%$; 5 mole \% $\mathrm{BG}$.

The dashed line indicates the limits within which some samples showed the presence of two lamellar phases.

Fig.11. Variation of the d spacings of the lamellar phase in different concentrations of dextran solutions for: 10 mole $\%$ PG ; $10 \mathrm{~mole} \% \mathrm{PI} O$ and 10 mole\% Na-oleate $\square$.

The dashed line indicates the limits within which some samples showed the presence of two lamellar phases. 


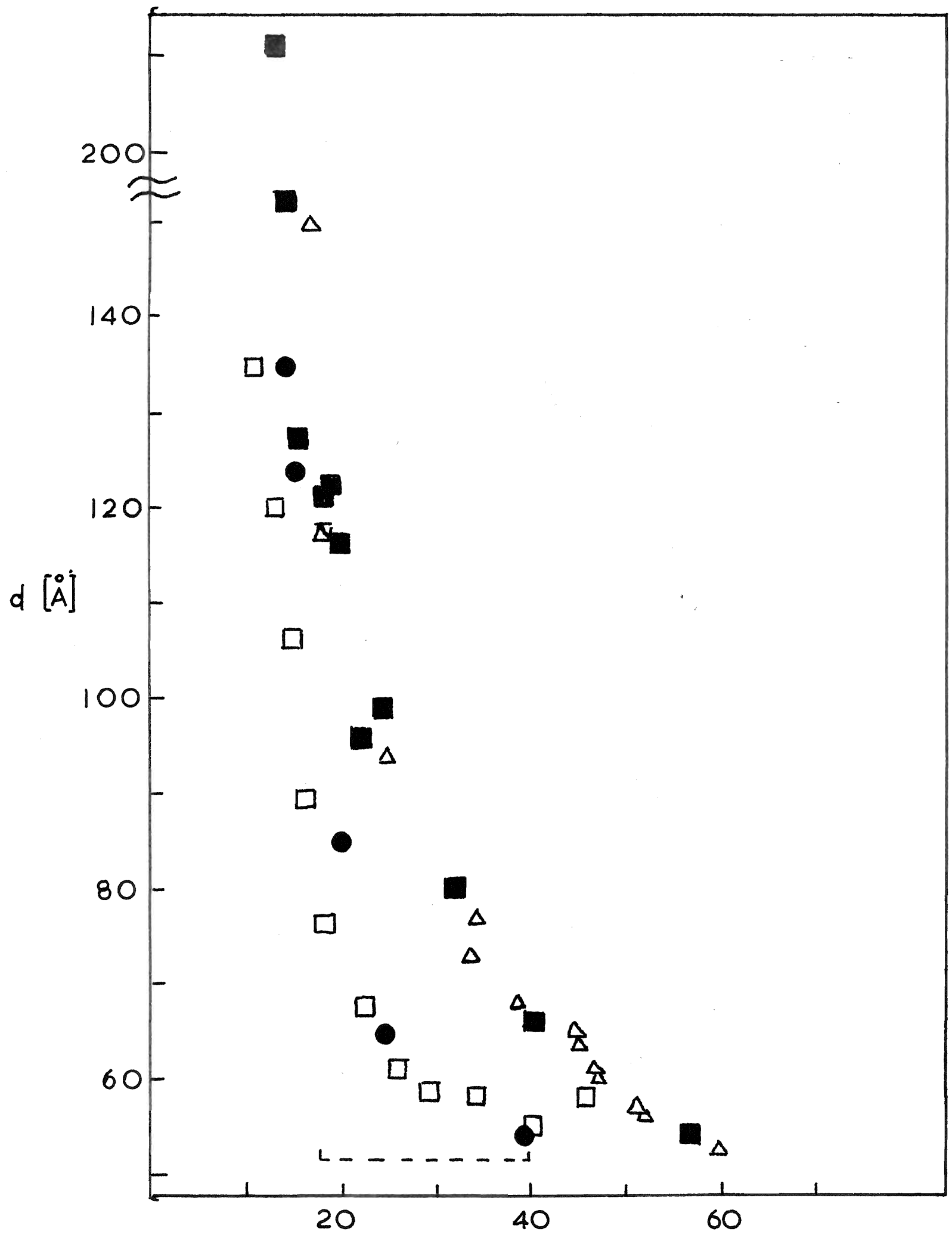

weight \& dextran

FIG. 10 . 


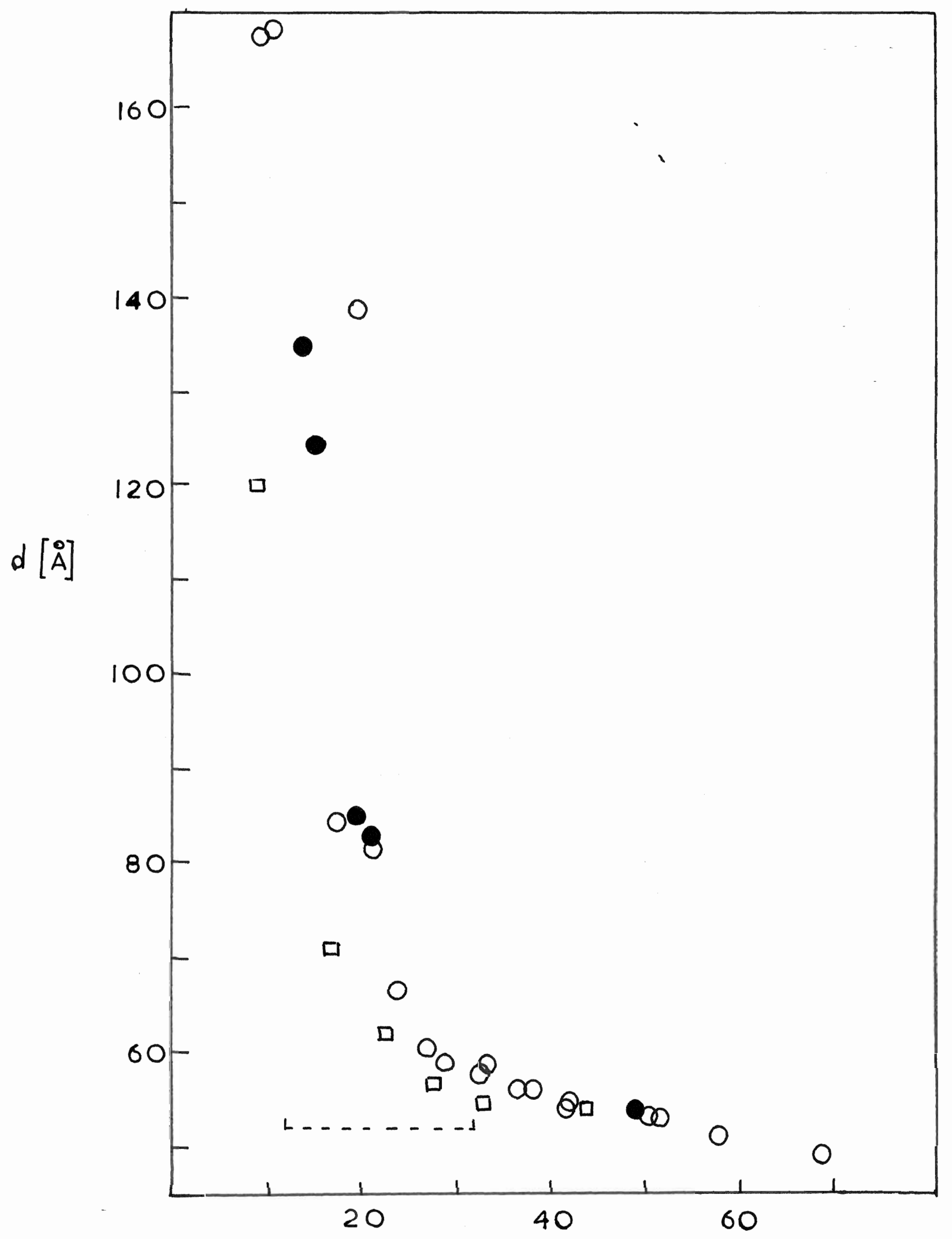

weight $\%$ dextran

$F|G|$.1 . 
water was allowed to flow freely across the membrane,

(iii) lipid was packed into a pressure cell and a known pressure was applied. The water was allowed to flow across the dialysis membrane-into the sample against this pressure.

Methods (i) and (ii) gave the same d spacings for small and medium swellings. For large d spacings of $100 \mathrm{~A}$ or more there seemed to be a disorder in the diffraction patterns due to penetration of the interbilayer spaces by dextran nolecules. In this region method (i) no longer gave consistent results. Method (iii) gave accurate results at high pressures, however at low pressures the inertia in the movement of the oiston in the pressure celI was the limiting factor in determining the accuracy. Using all three methods we were able to vary the external pressure in the range from $2 \times 10^{5}$ to over $1000 \times 10^{5} \mathrm{dynes} / \mathrm{cm}^{2}$.

When the curves obtained for different molar concentrations of $\mathrm{PG}$ in lecithin are compared (Fig.10), we see that for any given concentration of dextran higher d spacings resulted at higher bilayer charge density as expected. Also, it appears from Fig.11 that when the same percentage of charged lipid is used, the d spacings are very similar irrespective of the kind of lipid used. In both Figs 10 and 11 there is a range of cextran concentrations at which there appear to be two lamellar phases present. This double phase region was first observed in our studies of PI samples and later found with other 
charged linids. It seems to vary, in extent, with the lipid system studied but it appears consistently just above the maximum swelling of lecithin. The two phases do not arise as a result of separation of charged lipids from lecithin since the lower d spacing is higher than the maximum swelling of pure lecithin in excess water. There must exist a more complex separation of lipids which is not wholly understood but which has been observed by other workers as discussed in the Introduction.

2.3 The force curves for different lipid systems.

If we consider the chemical potential of water between lipid bilayers which is in equilibrium with the water in the bulk dextran solution, we recognize that it must be the same in both, as long as there is a free flow of water between them. In both cases the chemical potential of water is lower than that of bulk water. In dextran the lower chemical potential is caused by the lower concentration of water because of the presence of solute. In the lipid-water system, the lower chemical potential is due to reduced hydrostatic pressure arising from the net repulsive force between the bilayers. The lower potential. of water in dextran appears as an osmotic pressure while that between bilayers causes swelling and an increased d spacing. When the system is in equilibrium the osmotic pressure is a direct measure of the magnitude of the net repulsive force. To state it in terms of a force balance equation:

$$
\left|F_{\text {net repulsive }}\right|=\left|F_{E}\right|+\left|F_{R}\right|-\left|F_{A}\right|=\pi
$$


where symbols are the same as those used in the Introduction.

The osmotic pressure of the dextran solutions can be measured directly and accurately using a water or mercury column or pressure gauges, and such measurements were done in this laboratory by LeNeveu et al. (1976). Their results were used to construct the calibration curve of Fig.12. Figs 13,14 \& 15 give the plots of the net repulsive force F against appropriate $\mathrm{d}_{\mathrm{w}}$ values, which we call force curves. All force curves are very similar in shape and if one compares them for various concentrations of $P G$ in lecithin, higher concentrations of charge cause a shift of the whole curve upwards along the "Jog F" axis leaving the shape unchanged.

Each curve runs over three quite distinct force regions (Fig.17) and if one calculates the energy which is necessary to bring two lipid bilayers together one finds quite different results for these regions. Table VI shows the results calculated for the force curves of Figs $13 \& 14$, i.e. for 5,10 and 50 mole \% PG in lecithin (pure PG shows the same results as 50 mole \% PG). The energy can be calculated from the equation:

$$
\text { Energy }=\int_{d_{w_{1}}}^{d_{w_{2}}}\left(F_{H}+F_{F S}\right) d d_{W}
$$

where $F_{H I}=10^{11} \exp \left(-a_{W} / 1.93\right) \quad$ (IeNeveu et al.1976)

$$
F_{P S}=k T\left(2 \alpha / A d_{W}\right)(x / \tan X) \text { (Cowley et al.1978, }
$$

$\mathrm{d}_{\mathrm{w}_{1}}$ and $\mathrm{d}_{\mathrm{w}_{2}}$ are the initial and the final separations of the lipid bilayers during the movement for which the energy is calculated. 
Fig.12. Calibration curves for dextran solutions

a) Osmotic pressure of dextran solutions in $\mathrm{cm} \mathrm{Hg} \mathrm{(} \mathrm{values} \mathrm{taken} \mathrm{from} \mathrm{LeNeveu,} \mathrm{Rand} \mathrm{and}$ Parsegian, Nature,(1976) 259,601-603.).

b) Logarithm of osmotic pressure versus different concentrations of dextran solutions, calculated for the values of Fig.12 a. 


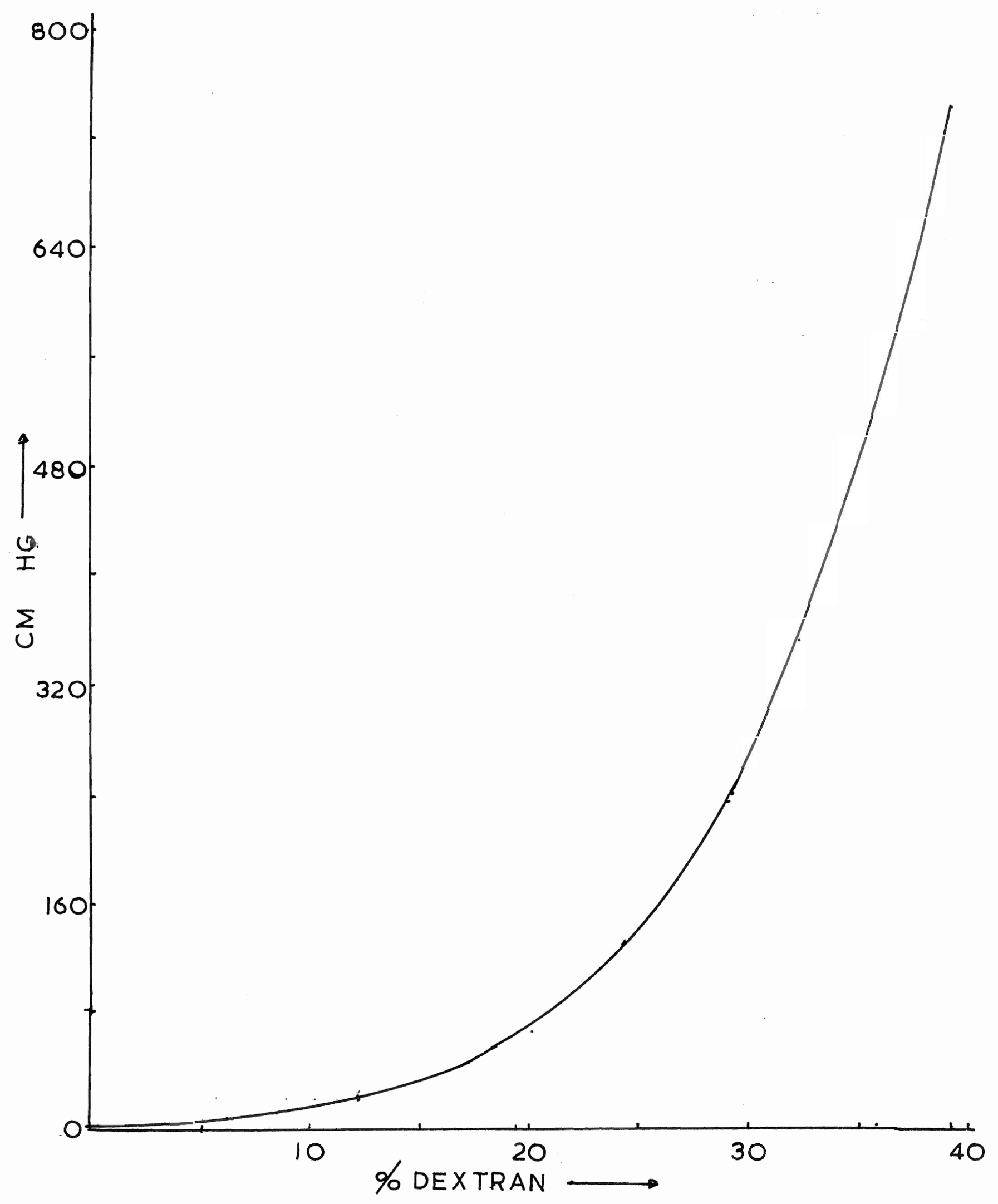

FIG.12a. 
46.

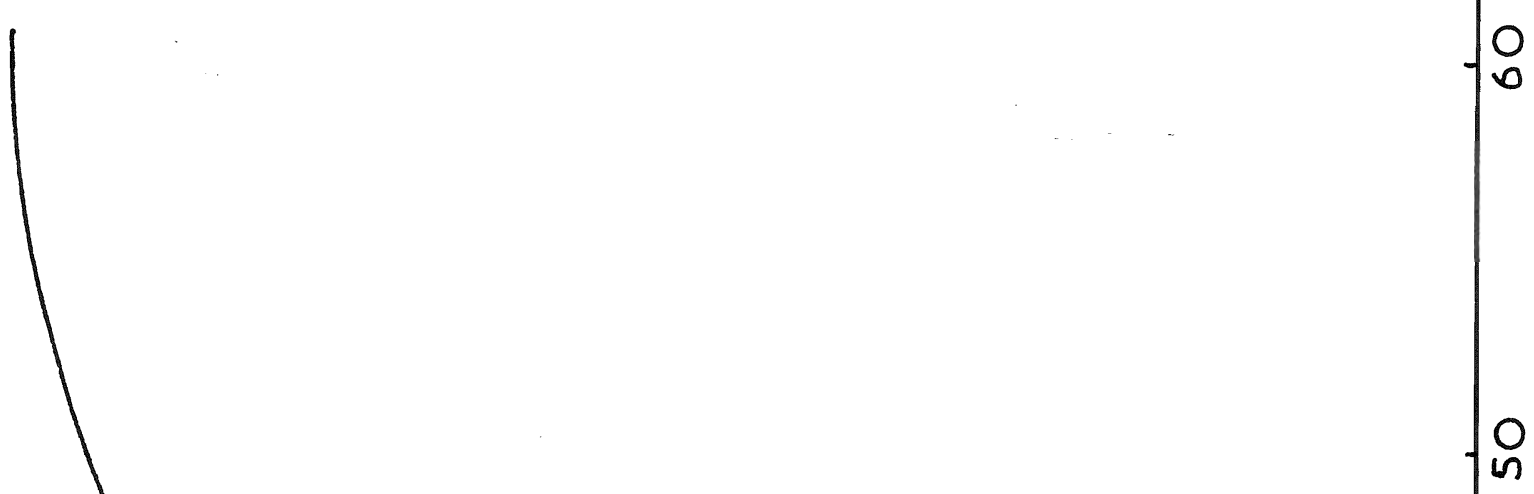

$F \mid G .12$ b. 
Fig.13. Relation between the net repulsive force F between phosphatidylglycerol- egg lecithin bilayers, and their separation $\mathrm{d}_{\mathrm{w}}$. The experimental points given are : 10 mole\% $\mathrm{PG} \bullet$ and $5 \mathrm{~mole} \% \mathrm{PG}$ 口. The solid lines give the theoretical net repulsive force for the areas per unit charge shown. Also marked are: the hydration repulsive force $\mathrm{F}_{\mathrm{H}}(-\cdots)$ and the electrostatic repulsive force $\mathrm{F}_{\mathrm{EIS}}(-\cdots)$.

Fig.14. Relation between the net repulsive force between lipid bilayers and their separation $d_{w}$ (as in Fig.13). The experimental points given are: $\Delta$ $100 \% \mathrm{PG}$ and 50 mole \% PG . The other focces marked are as in Fig. 13.

Fig.15. Relation between the net repulsive force between lipid bilayers and their separation $d_{W}$ * The experimental points given are: $10 \mathrm{~mole} \% \mathrm{PG}$ 10 mole $\%$ PI 0 and 10 mole $\%$ Na-oleate $\square$. The other forces are marked as in Fig. 13. 


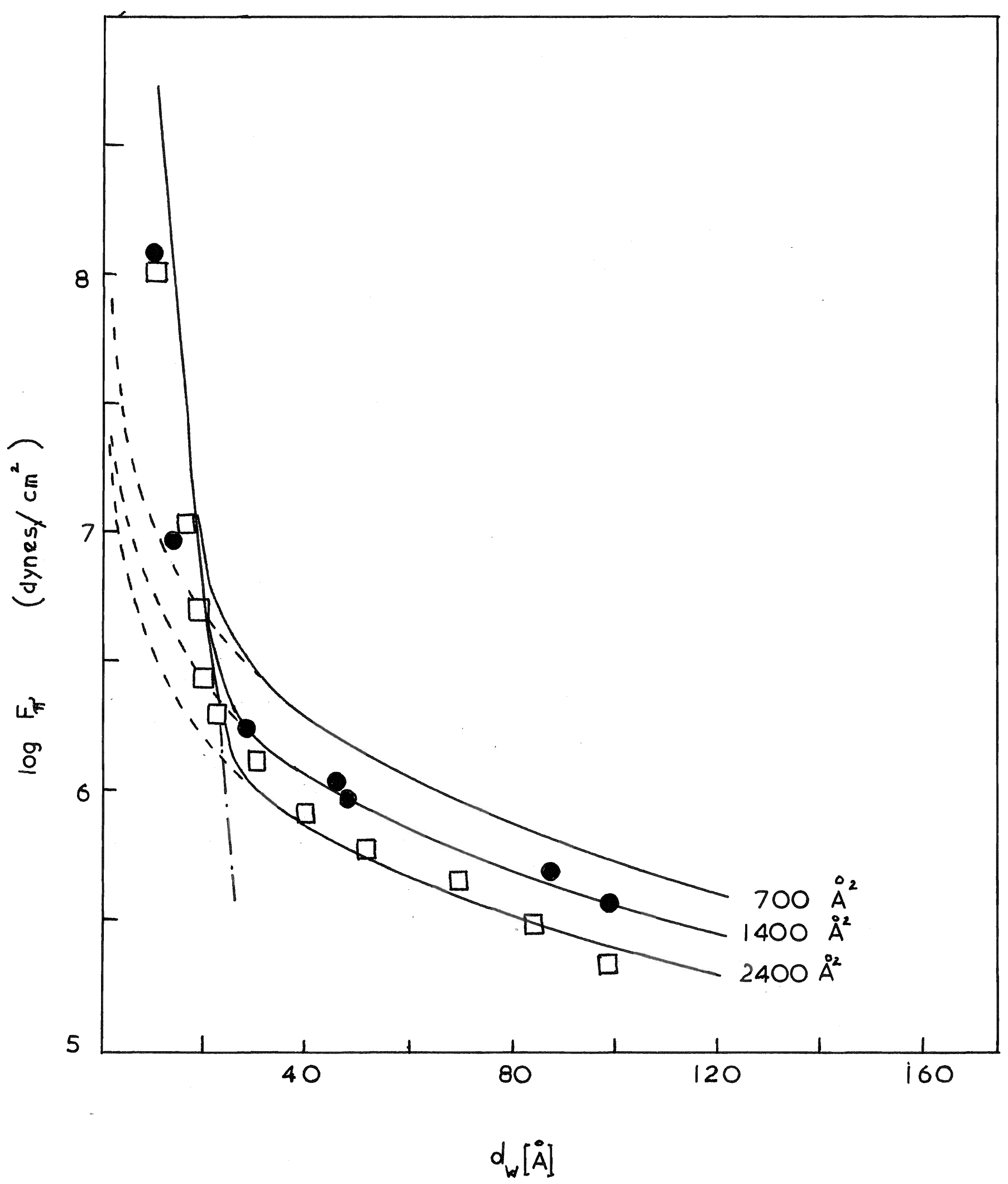

FIG.13. 
49.

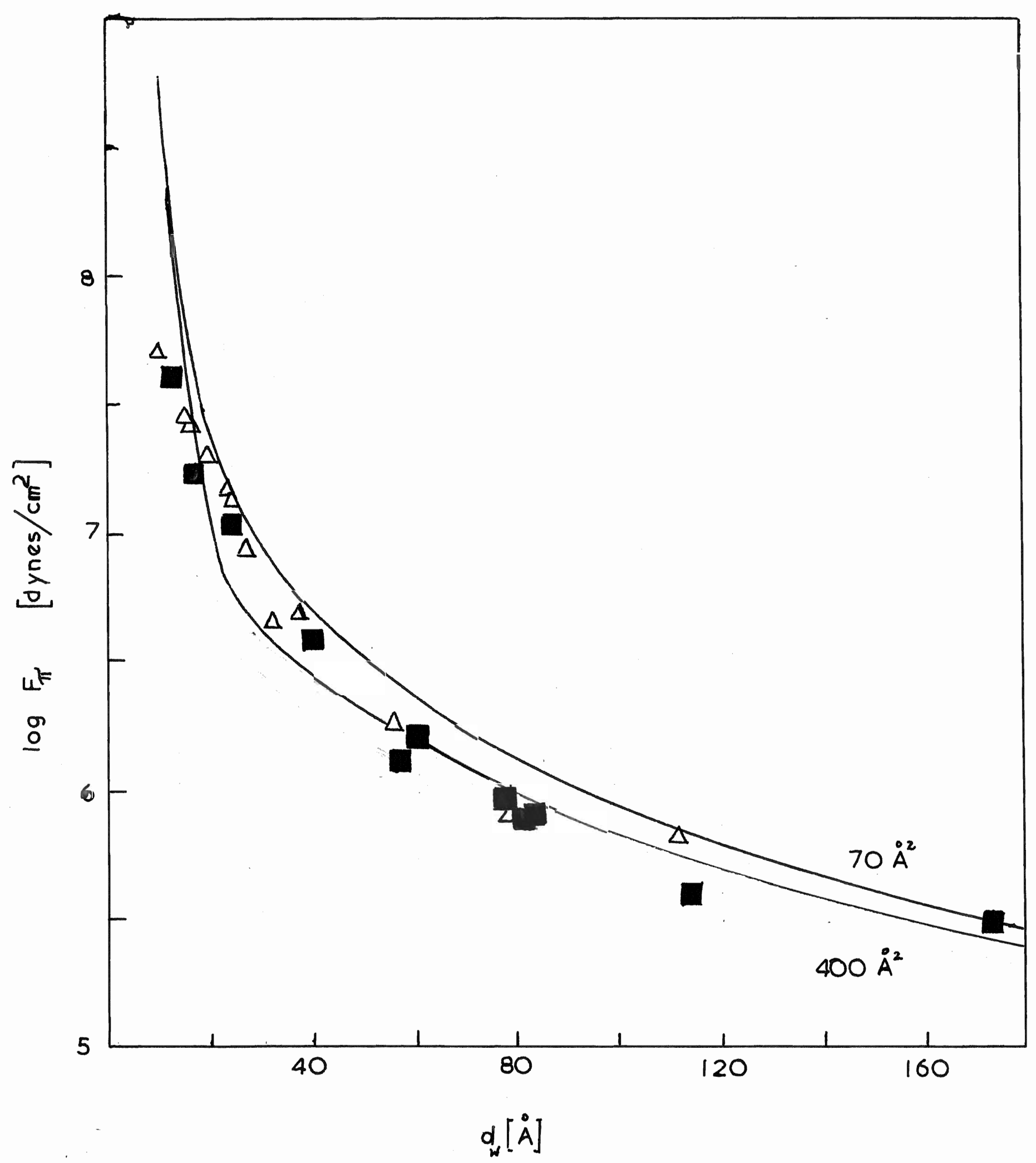

FIG. 14 . 


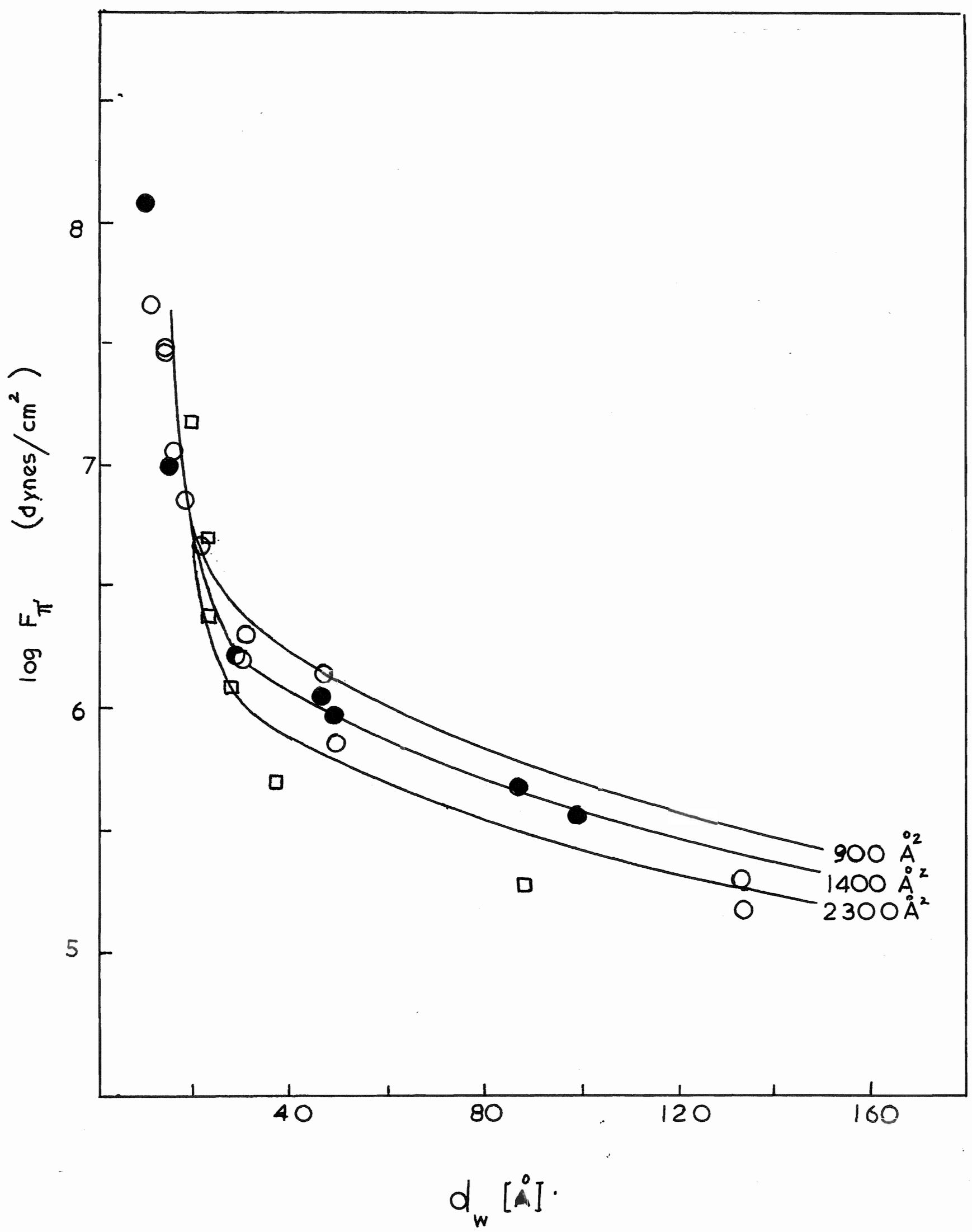

FIG. 15. 
For the present purposes sufficiently accurate estimates of the energy can be made if it is assumed that on the straight portions of the force curve the relation between pressure $P$ and bilayex separation $d_{W}$ is exponential,

$$
P=A \exp \left(-B a_{W}\right)
$$

where $A$ and $B$ are constants and

$$
\begin{aligned}
B & =\ln \left(P_{1} / P_{2}\right) /\left(d_{w_{2}}-d_{w_{1}}\right) \\
\text { Energy } & =\int_{d_{w_{1}}}^{d_{w_{2}}} P x \text { bilayer area } x d d_{W} \\
& =\text { bilayer area } x\left(P_{1}-P_{2}\right) / B
\end{aligned}
$$

Very large changes in pressure correspond to small changes in bilayer separation in region $I$ of the force curve, but the opposite is true in region II where very small pressure changes lead to substantial changes in bilayer separation. For example, when $d_{w}$ changes by $15 \AA$ in region II the pressure changes by less than $10^{5}$ dynes $/ \mathrm{cm}^{2}$. For this reason any residual pressure applied to the lipid in this region altered the measured d spacings substantially. We found that the packing of the samples into sample holders was very crucial when $d_{\text {w }}$ was greater than $40 \AA$.

In Figs 13,14 \& 15 both experimental (points) and theoretically predicted results (solid lines) are plotted. The theoretically derived equation used to calculate the total repulsive force between lipid bilayers is given in Appendix I. The best fit between experimental and calculated force curves was obtained in the following manner. First a series of theoretical force curves was generated by varying the values of $A / \alpha$ (the apparentarea per charge) 
in the equation for the total repulsive force. After these calculated curves were plotted the experimental points were introduced on the same graph. Then the region between the two calculated curves in which the experimental points were plotted was exponded by plotting a new series of calculated curves spaced close together. The final adjustment of $\mathrm{A} / \alpha$ value gave the calculated curve that went through most of the experimental points. Theoretical curves selected in this manner for different lipid systems are plotted in Figs 13, 14 \& 15. It was surprising to find that in order to obtain these best fitting curves the values for the charge density in the bilayers used in calculations were half or less of the density of $P G$ present in the prepared samples. In Table VII a list of the selected areas per charge for the best fitting theoretical force curves is given. This area per charge group can be expressed as the ratio $A / \alpha$, where $A$ is the area per charged group determined from the experimentally given composition of the lipid and $\propto$ is the fraction of ionized groups in the bilayer. Part B of Table VII gives the estimated values of $\propto$ expressed as the percent fraction of ions present to the total number of charged groups determined from the composition of different charged lipid-lecithin systems.

2.4 Effect of salt solutions and $\mathrm{pH}_{\text {. }}$

The presence of varying amounts of electrolyte in water changes the potential. of the double layer and causes collapse of the swelling induced by electrostatic repulsion. Fig.16 shows an example of such a collapsed swelling in 
Fje.16. Variation of the d spacing of lamellar phasein different concentrations of dextran solution for the lamellar phase of 10 mole\% Na-oleate with salt present in the dextran solutions. The concentrations of the salt solutions used: $\times 0.1 \mathrm{MNaCl} ; \Delta 0.01 \mathrm{M} \mathrm{NaCl} ; \cdot 0.1 \mathrm{M} \mathrm{NaCl}$ and $0.1 \mathrm{M} \mathrm{Na} \mathrm{HPO}_{4}$. Points belonging to the second phase are encircled. Two lines are sketched through the noints belonging to two phases. 

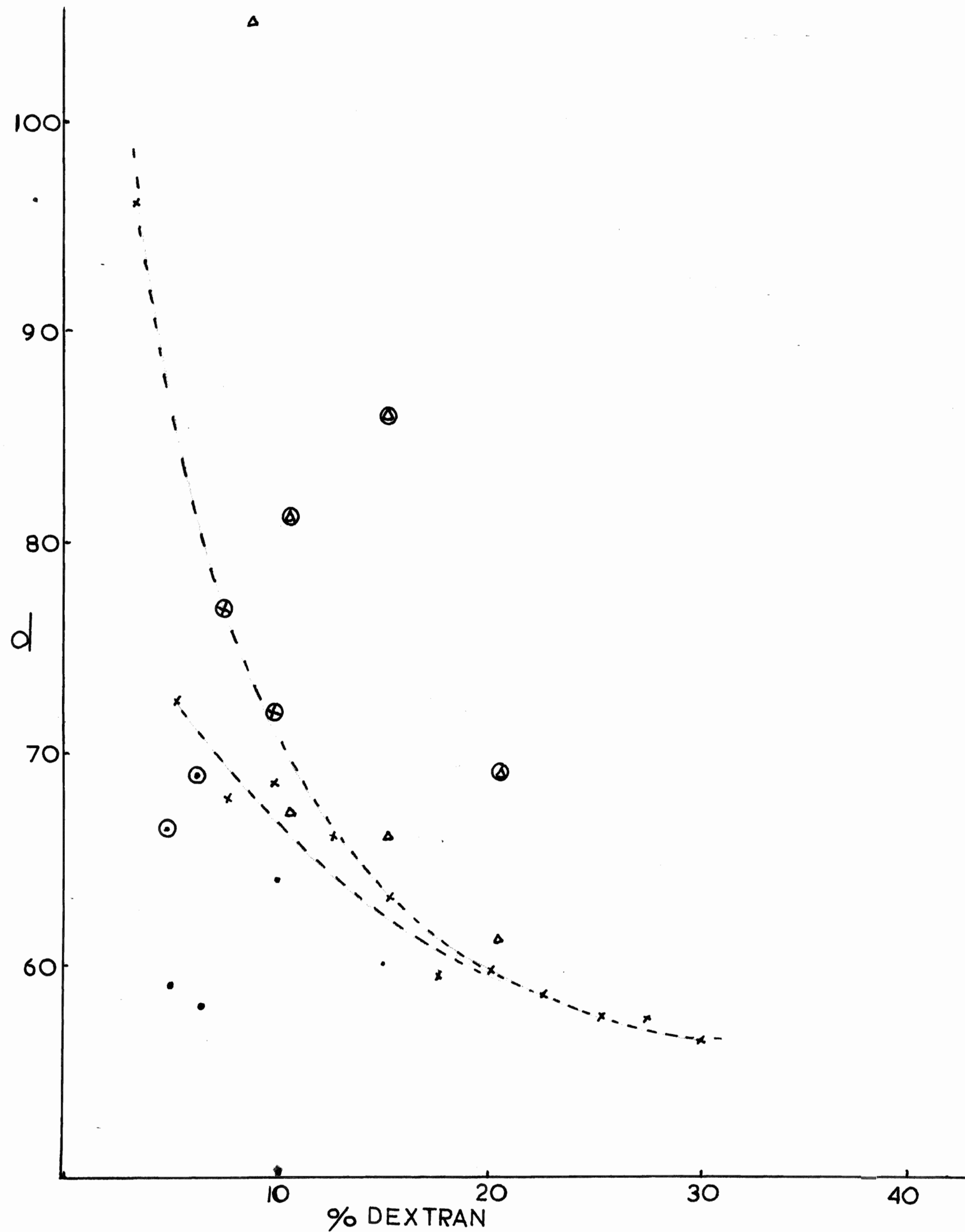

FIG. 16 . 
the 10 mole\% Na-oleate lecithin systen. Here the normal swelling of 10 mole\% Na-oleate in dextran solution of different concentration is compared to the swelling of the same lipid when the dextran solutions contain different amounts of $\mathrm{NaCl}$. The general shape of the curves represen-: ting the increase in the d spacings with decreasing dextran concentration is the same when the molarity of NaCl in dextran solutions is zero, $0.01 \mathrm{M}$ or $0.1 \mathrm{M}$. The two lamellar phases appear at about $23 \%$ dextran in the case of solutions with no salt and $0.01 \mathrm{M}$ in $\mathrm{NaCl}$, at the higher ionic strengths the second phase was observed at much 1ower dextran concentrations. There is a possibility however, that the lack of resolution prevented detection of the second phase at higher dextran concentrations. The observed phase separation was at first thought to be an outcome of the presence of some impuity or doubly ionized $\mathrm{Ca}^{\text {?t }}$ ions (section 1.2). The analysis of our dextran samples showed that the maximum amount of calcium present was below 1 ppm by weight. Dextran solutions with very low concentration of $\mathrm{CaCl}_{2}, 75 \mu \mathrm{M}$ and $750 \mu \mathrm{M}$, were prepared to check effects of such low ionic concentrations of $\mathrm{Ca}^{2+}$ and as shown in Fig.12 the extent of swelling in Na-oleate-lecithin system was not affected by these solutions and they could not cause the observed formation of two phases.

The $\mathrm{pH}$ of the unbuffered dextran solutions studied was 5.8 to 6.2 and it was thought that the low dissociation of the counter jons from the head groups of charged lipids could be improved by using buffered solutions. In 
Fig.16 results are plotted when $0.1 \mathrm{M} \mathrm{Na}_{2} \mathrm{HPO}_{4}$ buffer wa.s used in combination with $0.1 \mathrm{M} \mathrm{NaCl}$ in dextran of varying concentrations and equilibrated with the samples of 10 mole \% Na-oleate-lecithin. The over all result was a further collapse of d spacings as compared with $0.1 \mathrm{M} \mathrm{NaCl}$ solution only. Thus the addition of salts to produce buffered solution did not improve dissociation but had the effect of increasing the ionic strength of the solution with which the lipid was equilibrated.

Another way to ensure that the conditions in the bulk solution were such as to give complete dissociation of the phosphate groups of our phospholipids was by equilibrating samples of 10 mole \% PG with dextran solutions prepared at $\mathrm{pH} 6,7,8.2$ and 10.2 (Table VIII). AlI gave spacings similar to those obtained for PG samples equilibrated with dextran orepared in pure water. This rather surprising result could be explained by the possible difference between the ofH between bilayers of lipid and the pH of the bulk solution outside the lipid. For example, using electric double layer theory one can calculate the number of counter ions present in the interbilayer space. For 50 mole \% PG the bilayer separation $d_{W}$ is $60 \mathrm{~A}$ when $\log F_{\mathbb{J}}=$ 6.33, which gives the value for the concentration of counterions $n_{0}=0.5 \times 10^{20}$ (Appendix I). If all counter ions were $\mathrm{H}^{+}$this would give $\mathrm{pH}=4.1$ for the interbilayer space while the bulk pH of the dextran solution was 6 . In our case sodium salts of PG, PI and oleate were used and therefore the counter ions. in the interlamellar water were $\mathrm{Na}^{+}$, 
however because of the higher affinity of phosphate grouns for $\mathrm{H}^{+}$than for $\mathrm{Na}^{+}$the high proportion of $\mathrm{Na}^{+}$would be replaced by $\mathrm{H}^{+}$in the interbilayer space.

The partial dissociation observed for all our charged lipids and its independence of the $\mathrm{pH}$ of equilibrating solutions may be the outcome of the separation of lipids within the bilayer. If charged linids form domains within the matrix of the lecithin and the dissociation within each domain has a maximum, determined by geometry or packing, which is reached at a $\mathrm{pH}$ at about 4 , then the increase of pH above this value will not have any effect on dissociation. Also, if the dissociation within each domain is about $50 \%$ then the overall dissociation of the charged lipid lecithin system will also be $50 \%$. The separation of charged lipids into domains may also explain the presence of two phases as the charge in the bilayers would depend on the number of domains and this number may vary from bilayer to bilayer.

When the pH of the solution was made very low, 4.? and 3.6 as before no drastic changes in d spacings were observed (Table VIII). At pH of 3.6 which was the lowest pH studied, the lipid seemed to go through a phase change from the $I \alpha$ phase to a gel phase as indicated by the presence of frozen hydrocarbon chains in the diffraction photographs. 
3. DISCUSSTON OF RESUT,TS.

The equilibrium spacing of bilayers in contact with the dextran solutions results from the balance of four forces. Two of them, the hydration force $\mathbb{F}_{\mathrm{H}}$ and the electrostatic repulsion $\mathbb{F}_{F S}$ lead to repulsion between bilayers, and the other two, the van der Waals force $F_{A}$ and the externally apnlied osmotic pressure $F_{\mathbb{K}}$ tend to bring the bilayers closer together (see Introduction and Appendix I). The magnitude of each force varies with the bilayer separation $d_{w}$, and at equilibrium, the known external force $F$ is balanced by net internal force $\left(F_{H}+F_{F S}-F_{A}\right)$ which is dominated by a different single force in different ranges of $d_{w}$. The results plotted in Figs 13 to 15 give the relationship between the net repulsive force between bilayers and the separation $d_{W}$. All the curves have the same shape and each runs over three quite distinct force regions (Fig.17). In the first region, at very small separations where $d_{W}$ lies between 8 and $25 \AA$ the slope of the curve is quite steep, indicating fast changes of force with small changes in separation. This behaviour is very similar to that of pure lecithin, and when plotted together the two lines almost overlap as shown in Fig.17. This is the region where a strong repulsive force which we call the hydration force dominates the bilayer interactions. The second region is quite different. Here the slope is very shallow, and large changes in $a_{w}$ occur for small chan- 
Fig.17. The force curve for $5 \mathrm{~mole} \%$ PG showing three distinct regions with different rates of variation of the net repulsive force between lipid bilayers and their separation $d_{w}$. Region $I$ is dominated by the repulsive hydration force Region II is dominated by the electroststic repulsive force and Region III is a transition region between the two. Also shown are the two repulsive forces: the hydration force $F_{H}$ and electrostatic force $F_{E S}$ ( assumed to be positive and refer to the leit hand axis) and the attractive van der Waal's force $F_{A}$ (assumed to be negative and refers to the right hand axis). 


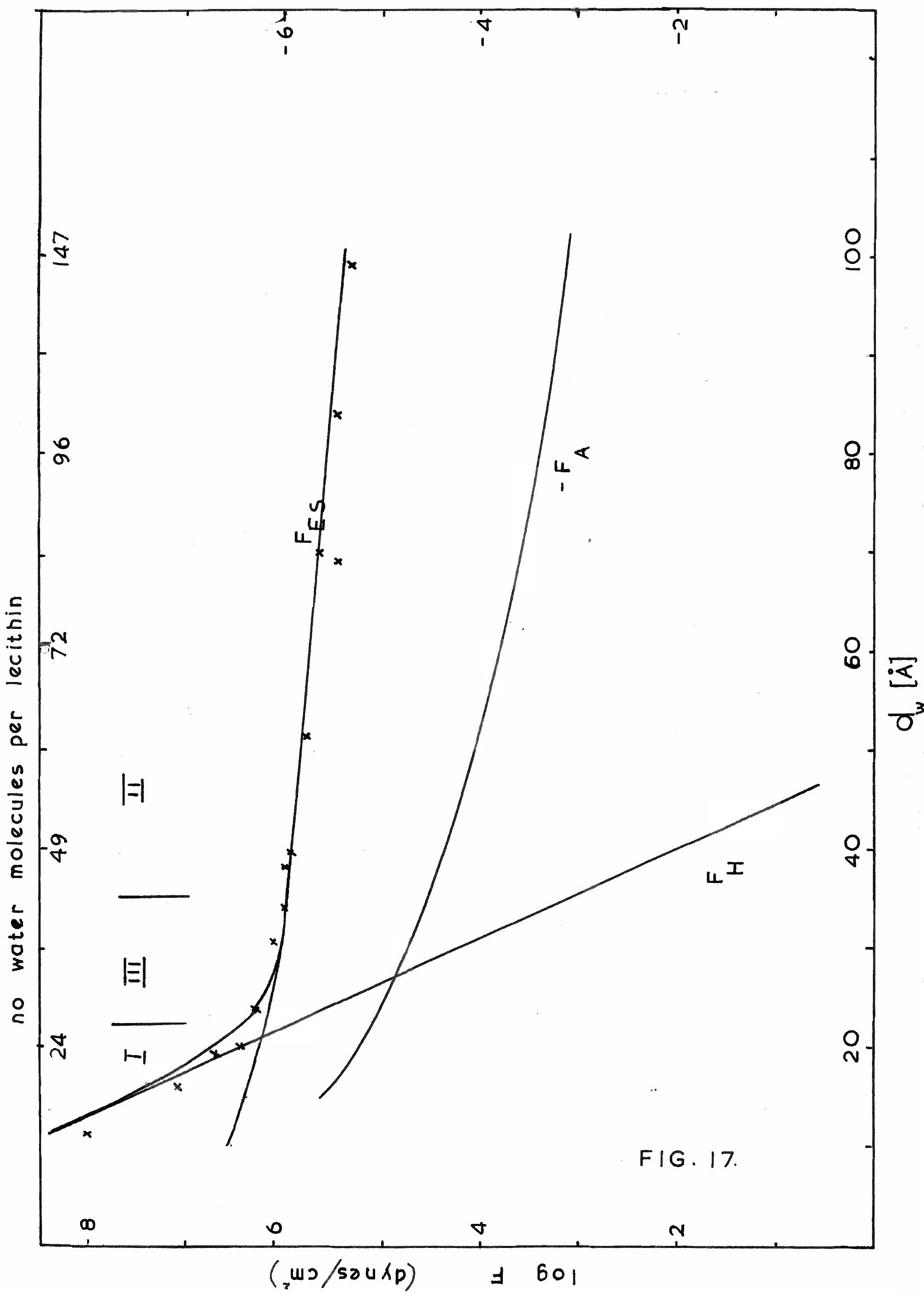


ges in force. This behaviour of the force curve is expected when dealing with the electrostatic repulsion between charged planar surfaces moving apart. This then is the region dorninated by electrostatic repulsion. The double layer theory gives an equation for the magnitude of this repulsion as:

$$
\mathrm{F}_{\mathrm{FS}}=\mathrm{kT}\left(2 \alpha / \mathrm{Ad}_{\mathrm{W}}\right)(\mathrm{X} / \tan \mathrm{X}) \text { Appendix } \mathrm{I}
$$

The third region 25 to $40 \AA$ is a transition region with the slope of the force curve changing very rapidly. The hydration force stjll dominates the interbilayer interactions at lower $d_{W}$ values and the electrostatic force takes over at larger values of $d_{w}$. In this region very often two phases were observed.

It was gratifying to find qualitative agreement between results calculated from double layer theory and those obtained experimentally. However, as shown in the Results, the best-fit force curves to the experimental points were obtained with areas per charge indicating that only $50 \%$ of the total number of $P G$ molecules present in lipid mixture (as expected from lipid composition) were dissociated. A recent monolayer study (Sacre \& Tocanne,1977) of synthetic $P G$ shows that its apparent pK varies around 5 and it depends on the salt concentration of the subphase, being higher at lower salt concentrations. When pure water was used as a subphase the PG was found fully protonated even at $\mathrm{pH}$ 6. Sacre \& Tocanne concluded that a high potential of $200 \mathrm{mV}$ at the surface of the charged lipid layer is capable of maintaining a very high proton and cation concen- 
tration near the surface. If such is the case then it is difficult to establish the relationship between the $\mathrm{pH}$ of the bulk solution with which the lipid is in equilibrium, and that near the charged lipid layer in the interbilayer space.

When fitting calculated force curves to the experimental points it was observed that $F_{\text {ES }}$ varies very slowly with surface charge density for double layers of high charge densities, and a change in area per charge by a factor of two was difficult to detect. However, at the lower charge densities $F_{\mathbb{E S}}$ was quite sensitive to the amount of charge and the difference between the curves for 5 mole\% and 10 mole\% PG in lecithin was definite in spite of some scatter of experimental points. The latter case showed convincingly that the lipid behaved as if not all of the available $\mathrm{PG}$ molecules were dissociated from Na-counter ions or protons. Parsegian (1974, Cowley et al.1978) argues that the incomplete charge dissociation may be only apparent, as the counterions may percolate behind the charged phosphate groups lowering the apparent charge density and changing the potential of the electrostatic double layer.

There is also the question of the lateral separation of lipids within the bilayer, which could have caused the phase separation in our experiments, and was observed by Luna et a..(1978) and Massari et a..(1977). Such a separation might have had some effect on the extent of dissociation of the counter ions. Also, our calculated results assumed that the charge in the bilayers was distributed 
uniformly, while if there was a lateral separation of lipids the charge could be arranged in areas of higher and Iower charge density.

The problem of two phases observed in our experiments has no satisfactory explanation. We lack systematic data as to the extent of the region in which two phases coexist and we were not able to establish any relationship between the spacings of the two phases. At the begining of our experiments it was assumed that the second phase was a spurious effect resulting from a faulty experimental technique and most of the effort went into elimination of this phenomenon rather than its study. There is another feature of the experimental results that needs a comment. The linid-water phase diagrams Figs 8 \& 9, showed that the head groun area for charged PG was smaller by $5 \AA^{2}$ from that of neutral PC. It was a surprising result as we expected the strong electrostatic repulsion to keep the molecules of PG as far apart as possible. A closer look at the hear group structure of the molecules, however, makes it less surnrising. The head group of PC molecule is much larger than that of PG molecule and without electrostatic repulsion one would expect even tighter packing for PG. For lecithin the maxjmum area per head group, $S_{\text {max }}$, was found to be $72.5 \AA 2$. and this corresoonds to an area swept by a freely rotating elongated groug $4.8 \AA$ long. If, however there is a prefered orientation for the choline groups, and each. groun cannot deviate by more than 45 degrees from the direction of this 
prefered orientation, then the measured area could accomodate a group more than 8.5A long. In the case of $\mathrm{PG}$ the $\mathrm{S}_{\mathrm{max}}$ is $67.5 \mathrm{~A}^{2}$ and this area could accommodate a rotating head. group 4.6 A long or a group with restrained motion (within 45 degrees from the prefered orientation. 8.? A long. But the head group of a $\mathrm{PG}$ molecule is smaller than the head group of a PC molecule and for unionised PG one would expect even smaller area per head group than that observed. It appears therefore, that in spite of a difference in the size of their head groups the distance between the phosphate groups is quite similar for $P C$ and $P G$. Such an equilibrium distance of about 8 A could result from a balance between repulsive electrostatic forces between $\mathrm{PO}_{4}^{-}$groups and strong van der Waals attractive forces between hydrocarbon chains. This attraction between hydrocarbon chains known as "hydrophobic forcel must play an important part in the packing of the molecules. The position of the diffraction line at 4.6 A stays unchanged with addition of water and the corresponding thinning of the lipid bilayer, indicating that the predominant distance between neighbouringchains stays unchanged. Thus when the addition of water increases the hydration of the charged groups and therefore increases the overall area per group, the hydrocarbon chains in their tendency to keep the average neighbour to neighbour distance unchanged would respond with increased rotation and generel orientation of the chains such as to facilitate neighbour to neighbour contacts, at least for parts of the chains, to stay at $4.6 \mathrm{~A}$. Such responce of the hydrocarbon chains to increased hydration of head groups would result in thinning of the lipid bilayer. 


\section{CONCLUSIONS.}

1. The net repulsive force between charged lipid bilayers was measured in a number of lipid systems. The curves showing the variation of force as a function of separation of the bilayers show, for all charged lipid system studied, three very different regions: In region I ( for $d_{W}<25^{\circ}$ ) the hydration force is predominant and there are large changes in force with small changes in separation of bilayers; in this region considerable energy is necessary to bring two lipid bilayers closer by a few angstroms (e.g. 9kT units have to be expended to move two faces of area $10,000 \mathrm{~A}^{2}$ from 20 to $15 \AA$ in the case of $5 \mathrm{~mole} \% \mathrm{PG}$ in lecithin).

In region II (for $\mathrm{d}_{\mathrm{w}}>40^{\circ} \AA$ ) the electrostatic forces predominate and the interactions are well described by the electrostatic double layer theory; here the force changes very slowly with large changes in bilayer separations and only small energies are required in changing separations of bilayers (e.g. $0.5 \mathrm{kT}$ units are required to bring two faces of area $10,000 \mathrm{~A}^{2}$ from 70 to $65 \AA$ separation for $5 \mathrm{~mole} \% \mathrm{PG}$ in lecithin). In region III ( for $25 \AA<\mathrm{d}_{\mathrm{W}}<40 \AA$ ) a transition between the two types of forces is observed, the slope of the force curve changes rapidly and the extent of this region is not well defined; in this region coexistance of two charged lipid phases was often observed. 
2. The variation of electrostatic interactions between charged lipid membranes, at large separations, is well described by the simple theory of the electrostatic double layer. The best fit of calculated curves to experimental points in region II was obtained when only $50 \%$ or less of the charged molecules present in the lipid bilayer were assumed to be dissociated.

3. The calculated electrostatic force curves show that at high surface charge densities even changes in area per charge as large as a factor of two, are difficult to detect because the electrostatic force FES varies slowIy with surface charge density. At Iow charge densities however, the FS for two charge densities differing by a factor of two can be clearly separated.

4. When the pil of the solution with which the lipid was in equilibrium was varied between 6 and 10 the change in dissociation among the charged linids was not detectable.

5. The importance of electrostatic forces in interactions of membranes at close approach seem minimal as the hydration forces overwhelm the electrostatic forces at separetions smaller than $25 \AA$. Screening of the electrostatic forces by small amounts of salt does not change the general shape of the force curves and though it will affect interactions between lipid bilayers in region II it will not lower the repulsive forces in region I, and therefore it should not facilitate approach or fusion of the membranes. 
67.

TABLES. 


\section{TABLE I}

Conductance measurements.

Solutions

Conductance
in mhos $\mathrm{cm}^{-1}$

Water tap water deionized water I $\begin{array}{ccc}\text { glass distilled water I } & 4.2 \\ \text { " } & \text { II } & 2.0\end{array}$ 26.5 3.8 4.2 1.1

Dextran solutions

$$
\begin{array}{r}
20 \% \\
5 \% \\
1 \%
\end{array}
$$

$$
\begin{array}{r}
90.0 \\
44.5 \\
8.5
\end{array}
$$

Deionized dextran solutions $20 \%$

$5 \%$

$$
\begin{array}{r}
23.0 \\
9.5
\end{array}
$$

$\mathrm{CaCl}_{2} \cdot 2 \mathrm{H}_{2} \mathrm{O}$ solutions

$$
\begin{array}{ll}
75 & \mathrm{l} \\
750 & \mathrm{M}
\end{array}
$$$$
23.5
$$$$
174.0
$$

Sanple I and sample II were run on two different days. 
Refractive indices of dextran solutions measured with Abbe refractometer.

Refractive index

$n$

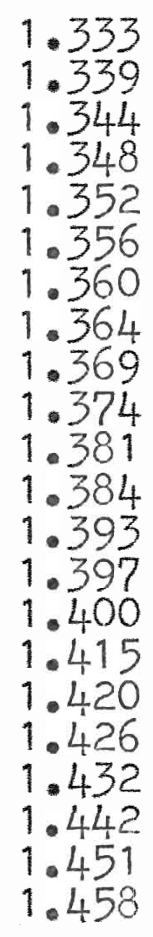

Concentration of dextran $\%$ by weight of dextran in solution

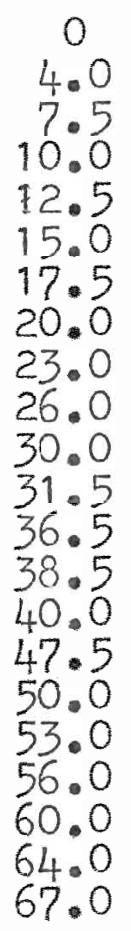


"d" spacings for Na-oleate lecithine system swelled in dextran solutions containing salt.

\begin{tabular}{|c|c|c|c|c|c|}
\hline \multicolumn{2}{|c|}{ system } & $\%$ dextran & $\begin{array}{l}\mathrm{d} \\
\mathrm{A}\end{array}$ & $\mathrm{d}_{\mathrm{A}}$ & $\mathrm{d}^{\mathrm{W}}$ \\
\hline \multirow{3}{*}{\multicolumn{2}{|c|}{$\begin{array}{r}10 \% \mathrm{Na}-\mathrm{ol} \text { leate } \\
0.1 \mathrm{M} \mathrm{NaCI}\end{array}$}} & $\begin{array}{l}30.5 \\
28 \\
25.75 \\
23.2 \\
20.5 \\
18.0 \\
15.5 \\
13.0 \\
10.25\end{array}$ & \multirow{3}{*}{$\begin{array}{c}56.4 \\
57.5 \\
57.5 \\
58.7 \\
59.8 \\
59.4 \\
63.2 \\
66.1 \\
68.6 \\
(72) \\
68 \\
(76.8) \\
72.5 \\
95.1\end{array}$} & \multirow{3}{*}{$\begin{array}{l}35.4 \\
33.5 \\
33.5 \\
33.2 \\
32.8 \\
32.4 \\
32.7 \\
32.0 \\
32.1 \\
(32) \\
32.5 \\
(31.3) \\
31.5 \\
31.1\end{array}$} & \multirow{3}{*}{$\begin{array}{l}21 \\
24 \\
24 \\
25.5 \\
27 \\
27 \\
30.5 \\
34.1 \\
36.5 \\
(40) \\
35.5 \\
(45.5) \\
41 \\
64\end{array}$} \\
\hline & & 7.75 & & & \\
\hline & & $\begin{array}{l}5.5 \\
3.5\end{array}$ & & & \\
\hline \multirow{4}{*}{$10 \%$} & \multirow{4}{*}{$\begin{array}{l}\text { Na-oleate } \\
0.01 \mathrm{M} \mathrm{NaCl}\end{array}$} & 20.8 & \multirow{4}{*}{$\begin{array}{l}61.4 \\
(69)^{4} \\
66 \\
(86) \\
67.4 \\
(81.7) \\
104.8\end{array}$} & \multirow{4}{*}{$\begin{array}{c}32.5 \\
(32.1) \\
32.0 \\
(31.2) \\
32 \\
(31.4) \\
30.5\end{array}$} & \multirow{4}{*}{$\begin{array}{c}27.9 \\
(36.9) \\
34.8) \\
(54.8) \\
35.4 \\
(50.3) \\
74.3\end{array}$} \\
\hline & & $15 . ?$ & & & \\
\hline & & 11 & & & \\
\hline & & 9 & & & \\
\hline $10 \%$ & $\begin{array}{l}\mathrm{Na}-\mathrm{ol} \text { eate } \\
\mathrm{O} .1 \mathrm{M} \mathrm{NaCl} \& \\
0.1 \mathrm{M} \mathrm{Na} \mathrm{NPO}_{4}\end{array}$ & 6.5 & $\begin{array}{l}58 \\
(69)\end{array}$ & $\begin{array}{l}33.5 \\
(32.1)\end{array}$ & $\begin{array}{l}24.5 \\
(36.9)\end{array}$ \\
\hline $10 \%$ & $\begin{array}{l}\mathrm{Na}-0 \text { leate } \\
0.1 \mathrm{M} \mathrm{Na} \mathrm{HPO}_{4}\end{array}$ & $\begin{array}{r}15 \\
10 \\
5\end{array}$ & $\begin{array}{l}60 \\
64 \\
66.5 \\
(59)^{5}\end{array}$ & $\begin{array}{l}33 \\
32.5 \\
32.2 \\
(33.4)\end{array}$ & $\begin{array}{l}27 \\
31.5 \\
34.3 \\
(25.6)\end{array}$ \\
\hline
\end{tabular}

Dextran solutions prepared as weight \% dextran in solution. 


\section{TABLE IV}

Structural parameters of the lamellar nhase

of lecithin and lecithin - charged lipid systems.

1. Lecithin

$\begin{array}{lcccc}\text { \%lipid } & d & d_{I} & d_{w} & s \\ \text { in water } & & & & \\ 91.7 & 49.3 & 45.2 & 4.1 & 58.4 \\ 87.8 & 49.6 & 43.5 & 6.1 & 60.7 \\ 81.6 & 51.0 & 41.6 & 9.4 & 63.5 \\ 73.2 & 53.6 & 39.2 & 14.4 & 67.5 \\ 66.7 & 55.9 & 37.3 & 18.6 & 71.2 \\ 62.9 & 59.7 & 37.6 & 22.1 & 70.6 \\ 58.9 & 61.8 & 36.4 & 25.4 & 73.0 \\ 55.0 & 61.4 & & & \\ 52.8 & 62.8 & & & \end{array}$

2. Phosphatidylglycerol in lecithin

a. $5 \% \mathrm{PG}$

$\%$ Iipid

in water

d

$d_{1}$

dw

S

94.8

88.9

85.0

79.9

75.0

70.0

59.8

$51 \cdot 5$

38.4

$\begin{array}{ll}49.8 & 47.2 \\ 50.0 & 44.5\end{array}$

50.5

51.0

42.9

52.6

54.7

62.9

61.3

78.8

39.5

$38 \cdot 3$

37.6

$36 . ?$

40.6

32.4

63.0

29.0

$93.0 \quad 35.7$

35.4

2.6
5.5
7.6

10.3

13.1

16.4

25.3

24.6

38.2

30.6

61.0

$57 \cdot 3$

109

31.6

77.4

b. $10 \% \mathrm{PG}$

$\begin{array}{lrlll}80.0 & 51.3 & 41.0 & 10.3 & 63.9 \\ 58.4 & 53.0 & 36.8 & 26.2 & 71.2 \\ 39.8 & 88.5 & 35.2 & 53.3 & \\ 30.1 & 92 & 36.6 & 55.4 & \end{array}$


TABLE IV (cont.)

c. $50 \% \mathrm{PG}$

$\begin{array}{lcccc}\text { \%lipid } & d & d_{I} & d_{w} & s \\ \text { in water } & & & & \\ 80.0 & 52.8 & 42.2 & 10.6 & 60.3 \\ 70.0 & 57.9 & 40.5 & 17.4 & 63.0 \\ 40.0 & 95.7 & 38.3 & 57.4 & 67.1 \\ \begin{array}{c}\text { 2. } 100 \% \text { PG } \\ 59.8\end{array} & & & & \\ 50.0 & 76.7 & 39.9 & 26.8 & 64.1 \\ & 77.8 & 38.9 & 38.9 & 66.6\end{array}$

3. Phosphatidylinositol in lecithin $10 \%$ PI.

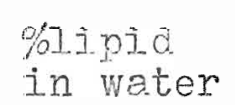

89.0
85.6
81.3
75.3
69.1
64.1
55.3
48.4
48.1
46.0
40.3
37.1
34.8
29.0
24.7 d.

$\begin{array}{ll}49.2 & 43.8 \\ 49.9 & 42.7 \\ 51.5 & 41.9 \\ 52.6 & 39.6 \\ 55.1 & 38.1 \\ 59.7 & 38.3 \\ 66.0 & 36.5 \\ 74.8 & 36.2 \\ 75.4 & 36.2 \\ 82.2 & 37.8 \\ 91.0 & 36.7 \\ 92.6 & 34.4 \\ 107.0 & 37.2 \\ 122.5 & 35.5 \\ 120.3 & \end{array}$

$d_{w}$

5.4
7.2

9.6

13.0

16.0

21.4

$29 \cdot \frac{5}{38}$

39.2

$44 \cdot 4$

$54 \cdot 3$

58.2

69.8

87.0

4. Na-oleate in lecithin

a. $5 \% \mathrm{Na}-01$

$\begin{array}{lllll}\text { \%ipid } & d & d & d & s \\ \text { in water } & & & & \\ 81.3 & 49.3 & 40.1 & 9.2 & 63.6 \\ 66.2 & 54.2 & 35.9 & 18.3 & 71.1 \\ 50.2 & 59.6 & 29.9 & 23.6 & 85.4 \\ 41.7 & 68.5 & 28.6 & 32.5 & 89.2 \\ * 95.2 & 50.3 & 47.9 & 2.4 & 53.1 \\ 90.0 & 50.3 & 45.3 & 4.0 & 56.1 \\ 85.1 & 50.4 & 42.8 & 7.6 & 59.4 \\ 80.1 & 51.6 & 41.3 & 10.3 & 61.6 \\ 77.4 & 52.1 & 40.6 & 11.5 & 62.6 \\ 75.0 & 53.0 & 39.8 & 13.2 & 63.9 \\ 70.1 & 55.6 & 39.0 & 15.6 & 65.2 \\ 64.8 & 59.3 & 38.5 & 20.8 & 66.0 \\ 60.0 & 63.5 & 38.1 & 25.4 & 66.7 \\ 52.2 & 69.2 & 36.1 & 33.1 & 70.4 \\ 50.0 & 73.5 & 36.7 & 36.8 & 69.3 \\ 39.7 & 82.5 & 32.7 & 49.8 & \\ 29.9 & 104.3 & 31.2 & 73.1 & \end{array}$




\section{TABLE IV (cont.)}

b. $6 \% \mathrm{Na}-01$

$\begin{array}{ccccc}\% \text { Iinid } & d & d & d & s \\ 81.3 & 50.6 & 41.1 & 9.5 & 62.1 \\ 78.5 & 51.0 & 40.0 & 11.0 & 63.8 \\ 55.7 & 58.3 & 32.5 & 22.3 & 78.5 \\ 39.0 & 85.5 & 33.3 & 49.5 & 76.6\end{array}$

c. $10 \% \mathrm{Na}-\mathrm{OI}$

$\begin{array}{lllll}69.7 & 51.8 & 36.1 & 15.7 & 68.5 \\ 66.1 & 54.7 & 36.2 & 15.5 & 68.3 \\ 57.6 & 57.0 & 32.8 & 24.2 & 75.3 \\ 55.6 & 59.3 & 33.0 & 26.3 & 74.9 \\ 55.4 & 61.3 & 34.0 & 25.3 & 72.7 \\ 50.7 & 67.0 & 34.0 & 31.0 & 72.7 \\ 47.8 & 64.8 & 30.9 & 28.6 & 80.0 \\ 45.5 & 66.4 & 30.2 & 30.4 & 81.8 \\ 41.3 & 82.5 & 34.1 & 46.5 & 72.5 \\ 40.7 & 75.2 & 30.6 & 39.2 & 80.8 \\ 35.1 & 92.9 & 32.6 & 56.9 & 75.8 \\ 30.8 & 94.0 & 28.9 & 58.0 & 85.5 \\ 30.0 & 97.6 & 29.3 & 61.6 & 84.3\end{array}$

All distances d are given in Angstroms and the areas available to each Iioid molecule $S$ in Angstroms square. indicates two phases present In 4a. * indicates the beginning of values measured by Mrs.N. Fuller. 


\section{TABLE V}

Variation of the d spacings of the lamellar phase as a function of osmotic pressure in three charged lipid

\section{systems.}

1. Phosphotidyl glycerol in lecithin

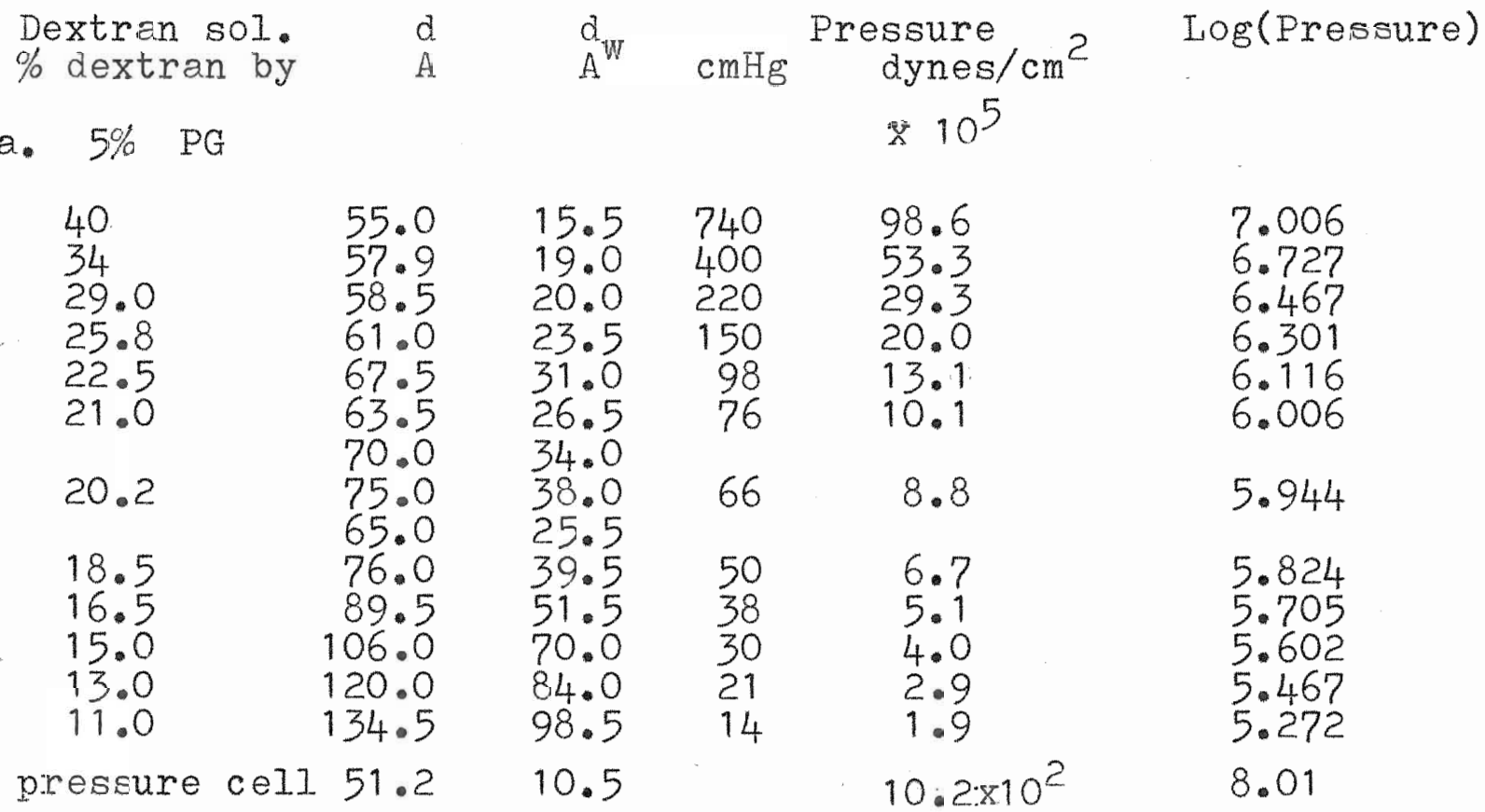

b. $10 \% \quad P G$

\begin{tabular}{|c|c|c|c|c|c|}
\hline $\begin{array}{l}39.3 \\
31.0 \\
24.6 \\
21.2 \\
20.0 \\
18.7 \\
17.5 \\
17.5\end{array}$ & $\begin{array}{c}54.0 \\
64.0 \\
64.8 \\
83.0 \\
85 \pm 2 \\
79.5 \\
105 \\
123\end{array}$ & $\begin{array}{l}15.0 \\
27.0 \\
28.3 \\
45.5 \\
48 \\
42 \\
85\end{array}$ & $\begin{array}{r}720 \\
284 \\
124 \\
80 \\
66 \\
50 \\
40 \\
40\end{array}$ & $\begin{array}{r}96.0 \\
37.9 \\
16.5 \\
10.7 \\
8.8 \\
6.7 \\
5.3 \\
5.3\end{array}$ & $\begin{array}{l}6.982 \\
6.578 \\
6.218 \\
6.028 \\
5.944 \\
5.927 \\
5.824 \\
5.824\end{array}$ \\
\hline $\begin{array}{l}15.5 \\
15.5 \\
12.9\end{array}$ & $\begin{array}{r}90 \\
124 \\
135\end{array}$ & $\begin{array}{l}52 \\
86 \\
98.5\end{array}$ & $\begin{array}{l}32 \\
32 \\
21\end{array}$ & $\begin{array}{l}4.3 \\
4.3 \\
2.8\end{array}$ & $\begin{array}{l}5.630 \\
5.630 \\
5.447\end{array}$ \\
\hline ssur & $51=$ & 10.0 & & $12.0 \times 10^{2}$ & 8.08 \\
\hline
\end{tabular}


TABLE V (cont.)

Dextran sol. weight

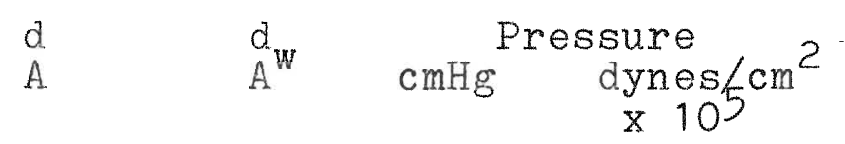

Log (Pressure)

c. $50 \%$ PG

56.5
45.5
40.0
31.7
29.2

*24. 0

*22. 0

$* 19.5$

18.5

*18.0

15.5

15.5

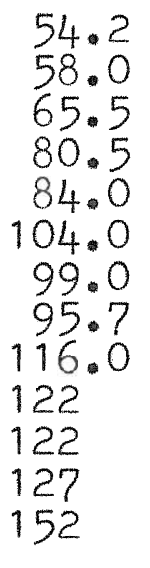

13.0

25.0

40.5

66.0

$51 \cdot 0$

57.5
78.0

84

84

89

114
760
310
224

$101 \cdot 3$

41.3

29.9

16.0

12.3

8.8

6.7

6.1

$4 \cdot 3$

$4 \cdot 3$
$7.58+$

$7.18+$

7.006

6.616

6.475

6.204

6.088

5.944

5.824

5.787

5.630

5.630

5.447

d. Pure PG

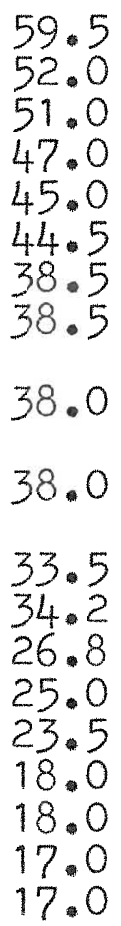

$$
\begin{aligned}
& 52.5 \\
& 56.0 \\
& 57.3 \\
& 60.4 \\
& 63.5 \\
& 65.0 \\
& 68.0 \\
& 71.0 \\
& 82 \\
& 69.0 \\
& 92 \\
& 69.5 \\
& 94 \\
& 73.0 \\
& (77) \\
& (85) \\
& 94.0 \\
& (91) \\
& (132) \\
& 117 \\
& 150 \\
& 146
\end{aligned}
$$

33.5

26.8

25.0

23.5

18.0

17.0

10.0

15.5

17.0

20.5

24.0

25.0

28.0

31.5

660

660

88.0

44

29.0

622

88.0

$7.69+$

$7.54+$

$7.51+$

$7.38+$

$7.34+$

$7.30+$

6.944

6.944

54

29.5 .622

82.9

6.919

82.9

6.913

6.854

$(338)$

536

71.5

53.9
$22 . ?$

18.0

$14 . ?$

(53)

(94)

79

112

108

6.1

6.1

8.8

8.8

6.731

6.355

6.255

6.166

5.787

5.787

5.727

5.727 
TABLE V (cont.)

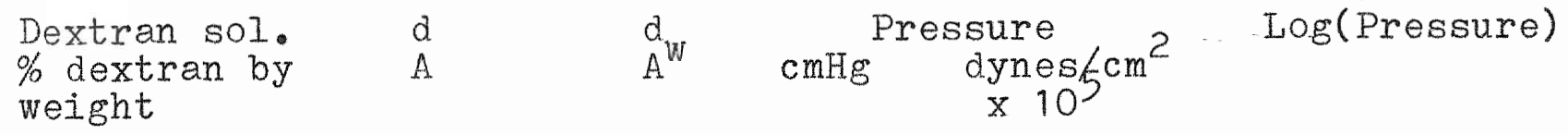

2. Phosphotidyl inositol in lecithine: 10\% PI

\begin{tabular}{|c|c|c|c|c|}
\hline $\begin{array}{c}* 68.9 \\
* 58.0 \\
* 52.0 \\
* 51.0 \\
* 42.0 \\
42.0 \\
38.3 \\
* 36.5 \\
* 33.5 \\
32.5 \\
29.0 \\
27.0 \\
* 26.0 \\
* 24.0 \\
20.0 \\
23 \\
* 19 \\
* 17.6 \\
15.5 \\
10.5 \\
9.0\end{array}$ & $\begin{array}{r}49.3 \\
51.4 \\
53.1 \\
53.3 \\
54.5 \\
55.0 \\
56.0 \\
56.5 \\
58.5 \\
57.7 \\
59.0 \\
60.6 \\
68.6 \\
66.3 \\
139 \\
81.5\end{array}$ & $\begin{array}{r}5.0 \\
10.5 \\
13.0 \\
13.0 \\
15.5 \\
16.0 \\
16.5 \\
16.5 \\
21.0 \\
19.0 \\
20.5 \\
23.0 \\
31.0 \\
29.5 \\
103 \\
46.0 \\
49.0 \\
101 \\
133 \\
132\end{array}$ & $\begin{array}{r}644 \\
536 \\
380 \\
342 \\
220 \\
172 \\
160 \\
120 \\
64 \\
104 \\
54 \\
42 \\
32 \\
16 \\
8\end{array}$ & $\begin{array}{r}85.9 \\
71.5 \\
50.7 \\
45.6 \\
29.3 \\
22.9 \\
21.3 \\
16.0 \\
8.5 \\
13.9 \\
7.2 \\
5.6 \\
4.3 \\
2.1 \\
1.1\end{array}$ \\
\hline
\end{tabular}

3. Sodium oleate in lecithin

a. $3 \% \mathrm{Na}-\mathrm{OI}$

$\begin{array}{rrrrrl}38.5 & 54.6 & 16.0 & 652 & 86.9 & 6.939 \\ 16.5 & 60.4 & 24.9 & 38 & 5.1 & 5.705 \\ 12.5 & 61.0 & 27.5 & 20 & 2.7 & 5.426 \\ 6.5 & 90.5 & 59.0 & & 0.53 & 4.72\end{array}$

b. $5 \% \mathrm{Na}-\mathrm{OI}$

$\begin{array}{llllll}42.0 & 54.5 & 19.5 & 860 & 114.7 & 7.06 \\ 37.5 & 55.5 & 20.9 & 596 & 79.5 & 6.900 \\ 33.0 & 56.5 & 22.0 & 360 & 48.0 & 6.681 \\ 27.0 & 57.5 & 23.5 & 172 & 22.9 & 6.360 \\ 21.8 & 60.0 & 27.0 & 88 & 11.7 & 6.069 \\ 16.8 & 65.0 & 33.0 & & & \\ & 64.0 & 31.6 & 40 & 5.3 & 5.727 \\ 11.5 & 85.5 & 54.3 & & & 5.328 \\ 6.0 & 70.0 & 38.4 & 16 & 2.1 & \\ & 110 & 79 & & & \\ & 124 & 93 & 4 & 0.53 & 4.724\end{array}$


TABLE V (cont.)

Dextran sol. $\%$ dextran by weight

c. $10 \% \mathrm{Na}-0 \mathrm{I}$

$$
\begin{aligned}
& 43.5 \\
& 32.5 \\
& 27.5 \\
& 22.5 \\
& 17.0 \\
& 11.0
\end{aligned}
$$

54.2

55.1

56.8

62.0

74.4

71.0

92.8

120

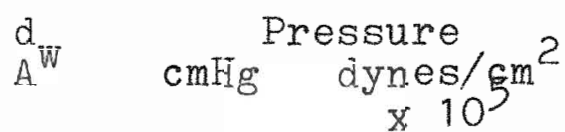

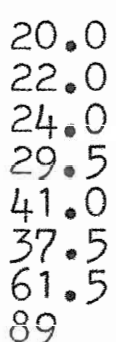

$\begin{array}{rr}342 & 45.6 \\ 184 & 24.5 \\ 98 & 13.1 \\ 40 & 5.3 \\ 14 & 1.9\end{array}$

Log (Pressure)

+ indicates values read from Fig. 6.b., all other values redd from Fig. 6.a.

* indicates samples swelling against dextran across the dialysis membrane.

()indicates weak lines or samples not fully at equillibrium 
Work necessary to bring two charged lipid planes together by distance $d_{w}$ calculated for different regions of force curve.

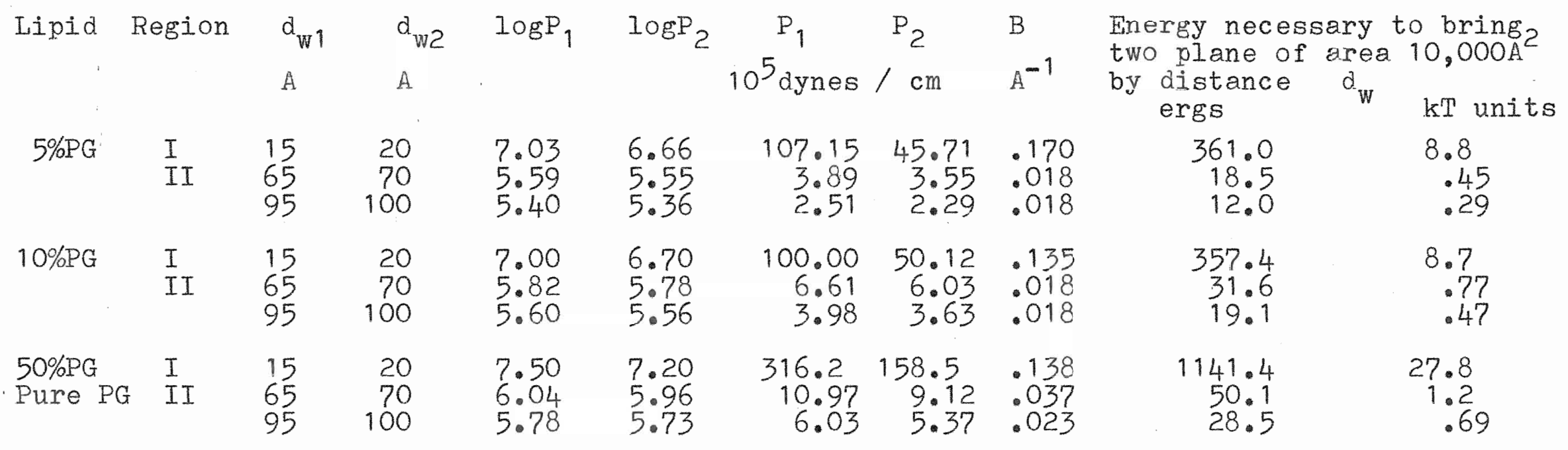

It is assumed that on straight portions of the force curve the relation between pressure $P$ and $d_{w}$ is exponential,

therelore

have

$P=A \exp \left(-B d_{W}\right)$ where $A$ and $B$ are constants. To evaluate $B$ we

$B=\ln \left(P_{1} / P_{2}\right) /\left(d_{w_{2}}-d_{w_{1}}\right)$
Energy $=\int_{d \omega_{1}}^{d \omega_{2}} P$ x Area $x d d_{w}=\operatorname{Area} \int_{d_{w_{1}}}^{\omega_{\omega_{2}}} \operatorname{Aexp}\left(-B d_{w}\right) d d_{w}=\operatorname{Area}\left(P_{1}-P_{2}\right) / B$ 
TABLE VII

An area per charged group in lecithin-charged lipid

bilayers and evaluation of the variable $\alpha$.

A. Comparison of measured and calculated results for the best fitting force curves, in region II

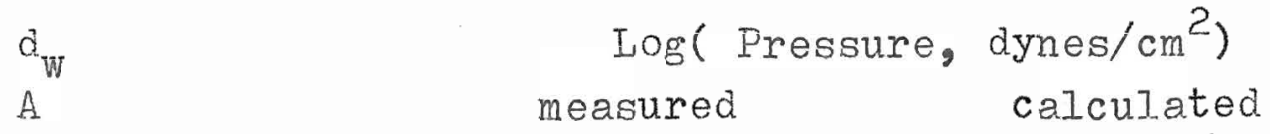

a. $5 \mathrm{~mole} \% \mathrm{PG}$, area per charge in calculations $2400 \mathrm{~A}^{2}$.
$39 \cdot 5$
70.0
84.0
5.824
5.705
5.602
5.467
5.272
5.883
5.755
5.601
5.506
5.420

b. 10 mole\% PG, area per charge used in calculations $1400 \mathrm{~A}^{2}$. 45.5 48.0 86.0 98.5

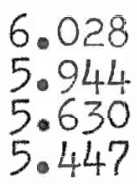

6.013

5.985

5.633

c. 50 mole\% PG, area per charge used in calculations $400 \mathrm{~A}^{2}$.
40.5
61.0
57.5
6.616
78.0
84.0
83.0
89.0
114.0
6.204
6.088
5.944
5.787
5.630
$5.630-5.447$
6.445
6.212
5.998
5.950
5.950
5.901
5.722

d. Pure PG, area per charge used in calculations $400 \mathrm{~A}^{2}$.

$$
\begin{array}{r}
38.0 \\
46.0 \\
56.0 \\
52.5 \\
79.0 \\
108.0 \\
112.0 \\
94.0
\end{array}
$$

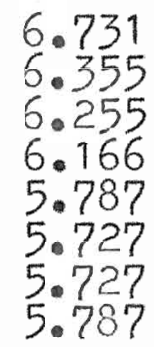

6.482

6.353

6.233

6.275

5.990

5.759

5.732

5.860 
TABLEVII (cont。)

$\begin{array}{ccc}\mathrm{d}_{\mathrm{w}} & \log \left(\text { Pressure, dynes } / \mathrm{cm}^{2}\right) & \\ \mathrm{A} & \text { calculated } & \mathrm{F}_{\mathrm{ESS}} \\ 1400 \mathrm{~A}^{2} & 2300 \mathrm{~A}^{2}\end{array}$

e. $10 \% \mathrm{PI}$

$\begin{array}{rlll}46.0 & 5.857-6.142 & 6.008 & 5.828 \\ 49.0 & 5.748 & 5.974 & 5.748 \\ 101.0 & 5.630 & 5.569 & 5.421 \\ 103.0 & 5.931 & 5.557 & 5.410 \\ 133.0 & 5.328 & 5.402 & 5.266 \\ 132.0 & 5.029 & 5.406 & 5.271\end{array}$

f. $\quad 10 \% \quad \mathrm{Na}-\mathrm{OI}$

$\begin{array}{llll}37.5 & 5.727 & 6.113 & 5.928 \\ 61.5 & 5.272 & 5.852 & 5.683 \\ 89.0 & 5.0 & 5.643 & 5.489\end{array}$

B. Evaluation of the variable $\alpha$, which gives the fraction of ionized groups in the bilayer.

\begin{tabular}{|c|c|c|c|c|}
\hline Lipid system & $\begin{array}{l}6 \text { of charged } \\
\text { ipid } \\
\text { measured) }\end{array}$ & $\begin{array}{l}\text { Assumed } \\
\text { area per } \\
\text { head group } \\
\mathrm{A}^{2}\end{array}$ & $\begin{array}{l}\text { Tstimated } \\
\text { A / } \\
\div \% \text { charged } \\
\dot{\dot{i}} \text { ipid }\end{array}$ & $\begin{array}{c}\alpha \\
\text { expressed } \\
\text { as \% of } \\
\text { ions* }\end{array}$ \\
\hline $\begin{array}{c}\text { lecithin }+P G \\
" 1 " \\
" 1 " \\
" 1 " \\
\text { Pure "PG } \\
" 1 " \\
" 1 \\
\text { lecithin + PI } \\
\text { Iecithin + Na-OI } \\
" ~\end{array}$ & $\begin{array}{r}5 \% \\
10 \% \\
50 \% \\
100 \% \\
10 \%\end{array}$ & $\begin{array}{l}68 \\
70 \\
68 \\
70 \\
63 \\
70 \\
63 \\
70 \\
63 \\
70 \\
63 \\
70 \\
70 \\
75 \\
70 \\
75\end{array}$ & $\begin{array}{l}2400 \\
2400 \\
1400 \\
1400 \\
700 \\
700 \\
400 \\
400 \\
700 \\
700 \\
400 \\
400 \\
2300 \\
2300 \\
1400 \\
1400\end{array}$ & $\begin{array}{l}56.6 \% \\
58.3 \% \\
48.6 \% \\
50.0 \% \\
18.0 \% \\
20.0 \% \\
31.5 \% \\
35.0 \% \\
9.0 \% \\
10.0 \% \\
15.8 \% \\
17.5 \% \\
30.4 \% \\
32.6 \% \\
50.0 \% \\
53.6 \%\end{array}$ \\
\hline
\end{tabular}

*\% of ions to the total number of the charged groups. 


\section{TABT. VIII}

\section{Rffect of $\mathrm{pH}$ on $d$ spacings of the lamellar phase of 50 mole $\%$ PG.}

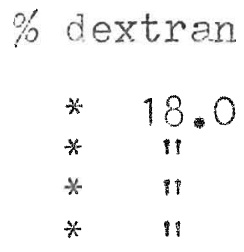

17.8
17.4
$\mathrm{oH}$

6.0

?.0

8.2

10.2

4.7
3.6 $d(1) A$

105.3

119.8

114.2

121.5

110.0

$102.0+$ d(2) A

128.6

132.1

123.0

126.0

* Measurements by Mrs.N. Fuller

+ Presence of diffraction line at $1 / \$=4.65$ A indicates formation of frozen chains.

$d(1)$ and $d(2)$ are two $d$ spacings measured for the same sample, $d(1)$ - after first equilibration, $d(2)$ - when the dextran solution equilibrating with the lipid was replaced with a new dextran solution (same concentration) and the sample was allowed to equilibrate again. 
REFERFNCES.

1. Abramson M.B.,Katzman R.o Gregor H.P.(1964) J.Biol. Chem., 239.

2. Barclay I.M.\& Ottewill R.H.(1970) Spec.Discuss. Faraday soc. 1, 138.

3. Barclay I.M., Harrington A.\& ottewill R.H.(1972) Kolloid Z.Z. Polym. 250, 655.

4. Boguslavskii I.I.,Krylov V.S.\& Shchipunov Yu.A. (1976) Biofizika 21(2), 266.

5. Brady G.W.\& Fein D.B.(1977)Biochim.Biophys.Acta464,249.

6. Bretscher M.S.(1972) Nature(London) New Biol.236, 46.

7. Casper D.I.D.\& Kirshner D.A.(1971) Nature(London) New Biol. 231, 46.

8. Castle J.D.\& Hubbell W.I.(1976) Bjochem.15, 4818.

9. Cowley A.C., Fuller N. L., Rand R.P.\& Parsegian V.A. (1978) Biochem. 17, 3163.

10. Dzaloshinskii I.E., Iifshitz R.M.\& Pitaevskii I.P. (1959) Adv.Phys. 10, 165.

11. Gingell D.\& Fornes J.A.(1976) Biophys.J. 16, 1131.

12. Griffin R.G., Powers I.\& Pershan P.S.(1978)Bjochem. $17,2718$.

13. Grodesky S.E.\& Marinetti G.V. (1973) Biochem. Biophys. Commun. 50, 1027.

14. Goulden P.T.(1976) J.Theor.Biol. 58(2), 425.

15. Gulik-Krzywicki T., Tardieu A.\& Luzzati V. (1969) Mol.Cryst.Liq. Cryst. 8, 258.

16. Gulik-Krzywicki T. (1975) Biochim.Biophys.Acta 415, 1.

17. Hauser H.,Phillips M.C., Tevine B.A.\& Williams R.J.P. (1976) Nature(London) 261, 390.

18. Israelachvili J.N.\& Adams G.F.(1976) Nature(London) 262, 774 .

19. Israelachvili J.N.8 Tabor D. (1972) Nature(London) $236,106$.

20. Janiak M.J., Small D.M.Q Shipley G.G.(1976) Biochem. $15,4575$.

21. Kantor H.I.\& Prestegrad J.H.(1978) Biochem. 17, 3592.

22. Kirschner D.A.\& Casper D.I.D. (1975) Proc.Natl.Acad. Sci. USA 72, 3513.

23. Kohler S.J.\& Klein M.F. (1977) Biochem. 16, 319.

24. Kosower M.M.,Kosower N.s.\& Wegman P. (1977) Biochim. Biophys.Acta $471,311$.

25. Isee A.G.(1977) Biochim.Bionhys.Acta 472, 237. 
26. Lelveveu D.M., Rand R.P.\& Parsegian V.A. (1976) Nature(London) 259, 601 .

27. IeNeveld D.M., Rand R.P., Parsegian V.A.\& Gingell D. (1977) Biophys.J. 18, 209.

28. Lelleveu D.M., Rand R.P., Gingell D.\& Parsegian V.A. (1976) Science 191, 399.

29. Levine Y.\& Wilkins M.H.F. (1971) Nature(Iondon) New Biol. 230, 69.

30. Lis I.J., Kauffman J.W.\& Shriver D.F. (1975) Biochim.Biophys.Acta 406, 453.

31. Luna F.J. \&McConnell H.M. (1977) Biochim.Biophys. Acta $470,303$.

32. Luzzati V. (1968) X-ray diffraction studies of linidwater systems, Biol.Membranes, ed.Chapman D., Acad. Press Inc. N.Y. 71-123.

33. Marcelja S.\& Radic N. (1976) Chem.Phys.Lett.42, 129.

34. Massari S.\& Pascolini D. (1977) Biochem.16, 1189.

35. McCinnell H.M., Devaux Ph.\& Scandelja C. (1972) Iatteral diffusion and phase separations in biological membrenes, Memb.Res., Acad.Press, 27.

36. McIaughlin Soix Harary . (1976) Biochem. 15, 1941.

37. McLaugh7in S.G.A. (1977) Current Topics Membrane Transport 9, 71.

38. McNenee M.G.\& McConnelI H.M. (1973) Biochem.12, 2955.

39. Michaelson D.M., Horowitz A.F.R KIein M.P. (1973) Biochem. 12, 2637.

40. Ninham B.W.2 Farsegian V.A. (1971) J.Theor.BinI.31,405.

41. Pagano R. ..8 Huang I. (1975) J.Cell Biol. 6?, 49.

42. Palmer K.J.\& Smith F.O. (1941) J.Cell.Comp.Physicl. $17,385$.

43. Papahadjopoulos D., Jacobson K., Nir S.\& Isac T. (1973) Bicchim.Biophys.Acte 311, 330.

44. Papahadjopoulos D., Hui S., Vai] W.T.\& Poste G. (1976a) Biochim.Bicphys.Acta $448,245$.

45. Papahajjonoulos T., Vail W.J., Pangborn W.A.\& Poste G. (1976b) Biochim. Biophys.scta $448,255$.

46. Papahedjopoulos D. , Poste G.,Schaeffer B. T.\& Vail W.J. (1974) Biochim.Biophys. Acte 352, 10.

47. Parsegian V.A. (1973) Annu.Rev.Biophys.Bioeng.2, 222.

48. Parsegian V.A.8 Minhar B.W. (1973) J.Theor.Biol.38, 101.

49. Parsegien V.A.\& Gingell D. (1973) J.Adhes. 4, 283.

50. Parsegian V.A. (1974) Ann.N.Y.Aced. 238, 362. 
51. Parsegian V.A. (1975) Iong range vander Waals forces Physical Chemistry: Mnriching Topics 4 , eds.

H. van 0lphen \& K.J. Mysels, Theorex, LaJolla Ca.,27.

52. Parsegian V.A. (1977) Soc.Neurosci.Sym.2, 161.

53. Poznanski M.\& Irange Y. (1976) Nature(Tondon) 259,420.

54. Rand R.P., Chapman D.8 I,arson K. (1975) Biophys.J. $15,1117$.

55. Rand R.P.8 Jugzati V. (1968) Biophys.J. 8, 125.

56. Rothman J.3.R Dawidowich T.A. (1975) Biochem.14, 2809.

57. Rothman J.F.\& Lenard J. (1977) Science 195, 743.

58. Sacre M.M.\& Tncanne J.E. (1977) Chem.Phys.Iipids 18,334.

59. Scandella C.J., Devaux Ph.\&e McConnell H.M. (1972) Proc.Nat.Acad.Sci.69, 2056.

60. Seeling A.\& Seeling J. (1975) Biochim. Biophys.Acte $406,1-5$.

61. Singer S.J.8. Nicolson G.I. (1972) Science 175, 720.

62. Singleton W.S., Gray N.S., Brown M.I.\& White J.L. (1965) J.Am.Oil Chem.Soc.42, 53.

63. Stoeckenjus W. \& Engelman D.M. (1967) J.Cell Biol. $42,613$.

64. Tabor D.8 Winterton R.H.S. (1969) Proc.Roy.Soc. A, $312,435$.

65. Tardieu A., Iuzzati V.\& Reman F.C. (1973) J.Mol.Biol. 75, 711.

66. Tocanne J.F., Ververgaert P.H.J.Th., Verkleij A.J.\& van Deenen I.I.M. (1974) Chem.\&Phys.Ijipids 12, 201.

67. Weinstein J.N., Yoshikami S., Henkart P., Blumenthal R. \& Hagins W.A. (1977) Science 195, 489.

68. Weinstein J.N., Blumenthal R., Sharrow S.O.\& Henkart P. (1977) Biophys.J. 17, 81a (Abstract).

69. Wilkins M.I.F., Blaurock A.E.\& Fngelman D.M. (1971) Nature(London) New Biol. 230, 72.

70. Mills M.C., Tinker D.0.\& Pinteric I. (1971) Biochim. Bionhys. Acta 241, 483. 


\section{DOUBTE LAYER THEORY.}

When charged lipid bilayers are placed in an aquaous solution of an electrolyte, an electric double layer is formed as a result of presence of excess of ions of one type on the surface of lipid and equivalent number of ions of opposite type in the aqueous solution near the interface.

According to the Gouy-Chapman treatment the ions in solution obey Boltzman distribution law:

$$
n_{i}=n_{\infty} \exp \left(-z_{i} e \psi / k T\right)
$$

where $n_{i}$ is number density of ions $i$ at a point having potential $\psi$

$n_{\text {m }}$ is number density of ions in the bulk solution

7 is the valence of the ionic charge.

The electrostatic potential $\psi=\psi(x)$ is found from the Poisson's equation:

$$
d^{2} \psi(x) / d x^{2}=-4 \pi \rho(x) / \varepsilon
$$

$\rho(x)$ is the charge density and $\varepsilon$ is the dielectric constant.

If we consider two planar surfaces separated by a distance $a_{W}$, the electrostatic potential $\psi(x)$ will vary along the line joining the surfaces as shown in Fig. 1A. The distance $x$ is measured from the midpoint between two plates.

The space charge density $\rho$ is the sum of ionic charges per unit volume, 


$$
\rho=\sum z_{i} e n_{i}
$$

The Poisson equation becomes:

$$
\frac{d^{2} \psi(x)}{d x^{2}}=-\left(\frac{4 \pi}{\varepsilon}\right) \sum Z_{i} e n_{\infty} \exp (-Z e \psi / k T)
$$

The boundary conditions for this equation are:

$$
\left.\frac{d \psi}{d x}\right|_{x=0}=0 \text { and }\left.\frac{d \psi}{d x}\right|_{x=\frac{d}{2} \omega}=-\frac{4 \pi \sigma}{\varepsilon}
$$

where $e$ is electrostatic charge and $\sigma$ is surface charge density in the lipid plane.

In our case the charge density in the lipid plane depended on the degree of dissociation of the lipid head groups and the only ionic species present in the interbilayer water space were the counter-ions that came off the lipid head groups in the process of dissociation plus ions arising from dissociation of water molecules.

The charge on lipid surface $\sigma$ is not assumed to be constant and continuous but it depends on the variable $\alpha$ which gives the fraction of ionized groups and is calculated self-consistently from the potential. The dissociation that takes place at the surface:

$$
\mathrm{AC} \rightleftharpoons \mathrm{A}^{-}+\mathrm{C}^{+}
$$

has a constant

$$
D=\left(\left[\mathrm{C}^{+}\right]_{\mathrm{S}}\left[\mathrm{A}^{-}\right]\right) /[\mathrm{AC}]=\left[\mathrm{C}^{+}\right]_{\mathrm{S}} /(1-\alpha)
$$

where $\mathrm{C}$ stands for counter-ions, and 


$$
\left[\mathrm{C}^{+}\right]_{\mathrm{S}}=\mathrm{C}_{\infty} \exp (-\operatorname{Re} \psi(\mathrm{x}) / \mathrm{kT})
$$

$\mathrm{C}_{\infty}$ being the concentration of counter-ions in bulk solution. Beceuse the lipid phosphate groups have stronger affinity for $\mathrm{H}^{+}$than for $\mathrm{Na}^{+}$(Tocanne et al. 1974) both cations must be present and it is assumed that $\mathrm{H}^{+}$is predominant.

The boundary conditions for Poisson equation are:

$$
\left.\frac{d \psi}{d x}\right|_{x=0}=\left.0 \quad \frac{d \psi}{d x}\right|_{x=d_{w / 2}}=-\frac{4 \pi e}{\varepsilon} \frac{\alpha}{A}
$$

Where $A$ is the area per dissociable phospholipid.

The charge density in the solution is:

$$
p(x)=e n(x)
$$

where $n(x)$ is number density of dissociated counterions, and according to the Boltzman law:

$$
n(x)=n_{0} \exp (-e \psi(x) / k T)
$$

we used here $n$ instear of $n$ o because in this case the bulk solution is pure water. The value of $n_{0}$ is found by integrating number density of counter ions from $\mathrm{x}=0$ to $x=d / 2$ and equating this integral to the density $\alpha /$ A of ionized grouns,

$$
\int_{0}^{d_{\omega / 2}} n(x) d x=\alpha / A=n_{0} \int_{0}^{d_{w / 2}} \exp (-e \psi(x) / k T) d x
$$

The solution to the Poisson equation for the case of linid swelling in a pure water was derived by Parsegian (Cowley et a. 1978) :

$$
\psi(x)=(2 k \pi / e) \ln |\cos (K x)|
$$

the above equation satisfies both the boundary conditions if $K$ fulfi.ls the relationship:

$$
\mathrm{Kd}_{\mathrm{W}} / 2 \tan \mathrm{Ka}_{\mathrm{W}} / 2=\left(\alpha \pi \mathrm{e}^{2} / \mathrm{kT}\right)\left(\partial_{\mathrm{W}} / \mathrm{A}\right)\left(\frac{1}{\epsilon}\right)
$$


(14) can be simplified by substituting $\mathrm{Kd} / 2$ by $\mathrm{X}$

$$
x \tan x=\left(\alpha \pi e^{2} / k T\right)\left(d_{w} / A\right)\left(\frac{1}{\varepsilon}\right)
$$

Tquation (13) can be rewritten

$$
\exp (e \psi(x) / x T)=\cos ^{2}(K x)
$$

and this when substituted into (12) gives

$$
\begin{aligned}
& n_{0} \int_{0}^{d_{\omega} / 2}\left[\cos ^{2}(K x)\right]^{-1} d x=n_{0} \int_{0}^{\frac{d w}{2}} 1 / K\left[\cos ^{2}(K x)\right]^{-1} d K x \\
&=n_{0} / K \tan (K d / 2)=\alpha / A \\
& \text { or } \quad n_{0}=(\alpha / A) K / \tan (K d / 2)
\end{aligned}
$$

What happens when dextran competes for water with the lipid? The osmotic pressure $\pi$ of dextran at the equilibrium must be equal to the pressure at the mid-point of aqueous solution between the bilayers, thus

$$
\pi=k \min
$$

and substituting for $n_{0}$ from (17) we get

$$
\begin{aligned}
& \pi=k T(\alpha / A)\left(K / \tan \left(\mathrm{Kd}_{W} / 2\right)\right. \\
& =k T\left(2 \alpha / \mathrm{Ad}_{W}\right)(\mathrm{X} / \tan \mathrm{X})
\end{aligned}
$$

Bquations (15) and (19) allow us to calculate the pressure between bilayers, which must arise from electrostatic repulsion, as van der Wals attraction is negligible for $\mathrm{d}_{\mathrm{W}}>27 \mathrm{~A}$. In the first step we find from equ. (15) the value of XtanX for given $d_{W}$ and $A$, then we evaluate $X$ and finally substitute this $X$ value into equ. (19) for the same $d_{W}$ and $A$ as in step one.

The following approximation was used to calculate X from $X \tan X$. 
Let $f=X \tan X$

then for $f<2$

$$
x=\sqrt{f-\frac{f^{2}}{3}+\frac{4 f^{3}}{45}-\frac{16 f^{4}}{945}+\frac{16 f^{5}}{14175}}
$$

for $f \geqslant 2$

$$
x=\frac{\pi}{2}\left(1-\frac{1}{1+f}+\frac{\pi^{2}}{12(1+f)^{3}}-\frac{\pi^{2}}{12(1+f)^{4}}-\frac{\pi^{4}}{72(1+f)^{5}}\right)
$$

The accuracy of equation (20) is to within $0.8 \%$ for $f=2$. and better for smaller $f$ values. For equation (21) the error is in the range of $0.7 \%$ for $f=2$ and smaller for all. larger if values.

The following program was used on a Wang computer:

10 Select $\mathrm{R}$

$20 \mathrm{C}=\log (10)$

$30 \quad \mathrm{E}=4.8 \mathrm{E}-10$

$e=4.8 \times 10^{-10}$ stat. coul.

$40 \quad K=4.1 \quad E-14$

$\mathrm{K}=\mathrm{kT}=4.1 \times 10^{-14}$ ergs

$50 \mathrm{E2}=80$

$\mathrm{Z} 2=\varepsilon=80$ for $\mathrm{H}_{2} \mathrm{O}$

$60 \operatorname{TINPUT} A, D$

A area in $\AA^{2}, D=d_{w}$ in $\AA$

$80 \mathrm{~A} 1=A^{*} 1 \mathrm{E}-16: D 1=D * 1 \mathrm{E}-8$ change A to $\mathrm{cm}$.

$90 F=H F I * F ! 2 * D 1 /(K * \$ 2 * A 1) \quad F=X \tan X$

100 IF F 2 THEN 130

$110 X=P I / 2\left(1-1 /(1+F)+P I ! 2 / 12(1+F)^{3}-P I ! 2 / 12(1+F)^{4}\right.$ $\left.-P I ! 4 / 72(1+F)^{5}\right)$

120 GO TO 140

$130 X=\operatorname{SOR}\left(F-F^{2} / 3+4 F^{3} / 45-16 F^{4} / 945+16 F^{5} / 14175\right)$

$140 \mathrm{Y}=\mathrm{TAN}(\mathrm{X})$

$150 Z=X / Y$

$\mathrm{Z}=\mathrm{X} / \tan \mathrm{X}$

$160 \mathrm{P}=2 * \mathrm{~K} * \mathrm{Z} /(\mathrm{A} 1 * \mathrm{D} 1)$

$\mathrm{P}=\pi=2 k \mathrm{kTX} / \mathrm{Ad} \mathrm{w} \tan \mathrm{X}$

170 PRINT A, D, F, X, Y, Z, P, I,OG(P)/C

180 GO TO 160

The values $A$ stand for $A / \propto$ and they were chosen to accomodate different levels of dissociation. The calculated values were plotted against $d_{w}$. 


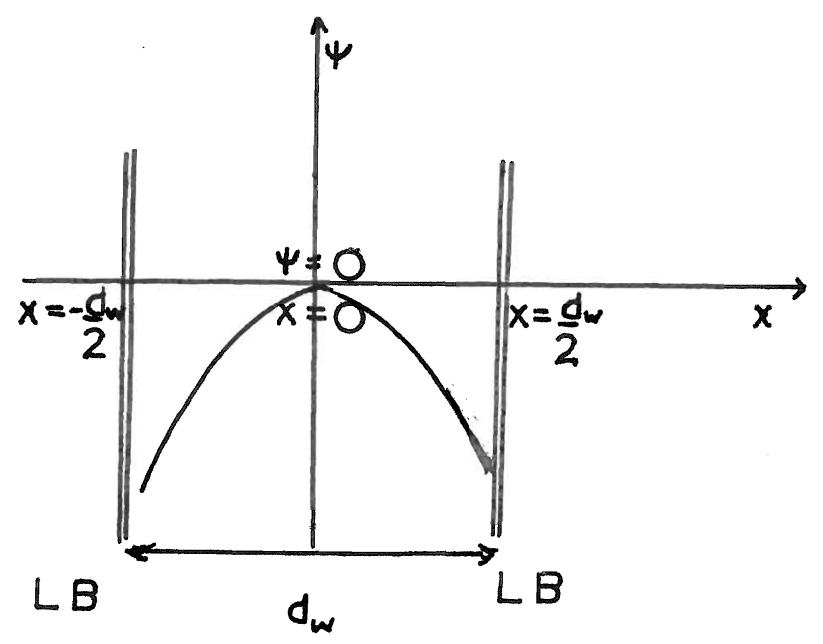

Fig. 1A. The variation of the electrostatic potential $\psi(x)$ between two surfaces of lipid bilayers (LB). The variable $x$ is the distance measured from mid point between the surfaces.

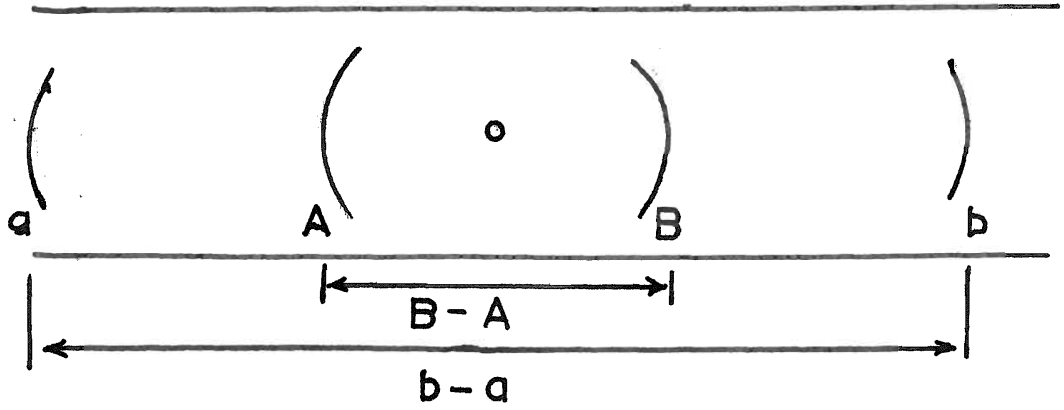

Fig. 2A. Simplified diffraction pattern showing single order of diffraction for lipid bilayer, lines $A$ and $B$, and for Teflon, lines $a$ and $b$. 


\section{APPENDIX II}

\section{CAIIBRATION OF X RAY PHOTOGRAPHS.}

To produce an internal standard for accurate measurements of diffraction lines on each X-ray photograph taken, a few miligrams of dry Teflon powder were included with most lipid samples studied. Such arrangement gave us a TefIon powder line on each film at $\theta=9^{\circ} 3^{\prime} 24^{\prime \prime}$ which corresponds to the d spacing of $4.87 \AA$. The d spacing for Teflon was found to be independent of temperature, from room temperature up to $40^{\circ} \mathrm{C}$. The original calibration of the TefIon line was done with the help of Berylium Acetate diffraction pattern. The crystallographic data for Berylium Acetate is well established, its symmetry is cubic with the spacing equal to $15.74 \pm .01 \AA$. In the original measurements Teflon tape instead of powder was used. A piece of tape was streched, in turns, across each of the two mica windows of the specimen holder and two photographs with superimposed diffraction patterns of Teflon and Berylium Acetate were taken. From these photographs the spacing of Teflon was found to be $4.87 \pm .01 \AA$.

To find a correct d spacing for the studied lipid bilayer from the photograph on which a Teflon line was recorded, the following measurements were made. The Fig.2A shows a simplified diffraction pattern with only a single order of diffraction for lipid bilayer (Iines $A$ \& $B$ ) and Teflon lines $a$ and $b$. It should be noted that a good $\mathrm{X}$-ray photograph would show a few orders of diffraction, 
sometimes five or six.

In order to find the diffraction angle $\theta$ for the given lipid line we can write an equation for $\theta$ using the symmetry of the camera:

$$
\theta=(B-A) / 8 R
$$

where $R$ is the radius of the camera, and can be determined from the measurement of the position of the Terlon line:

$$
R=(b-a) / 8 \theta_{\text {Teflon }}
$$

From Bragg equation we have:

$$
\theta_{\text {Teflon }}=\sin ^{-1}\left(\lambda / 2 \mathrm{~d}_{\text {Teflon }}\right)
$$

and we can write:

$$
\begin{aligned}
\theta & =(B-A) /(b-a) x \theta_{\text {Teflon }} \\
& =(B-A) /(b-a) x \sin ^{-1}\left(\lambda / 2 d_{\text {Teflon }}\right) \quad(4) \\
d & =\lambda / 2 \sin \theta=\lambda / 2 \sin \left((B-A) /(b-a) x \sin ^{-1} \lambda / 2 d\right)
\end{aligned}
$$

Since $\theta$ is very small we can replace in (5) $\sin \theta$ with $\theta$, introducing an error of less than tenth of a percent.

Thus $d=(B-A) /(b-a) x d_{\text {Teflon }}$

The total error in (6) is less than $1 \%$ for diffraction lines for lipid of second order or higher. The maximum error comes in the measurement of a small distance $B-A$. The typical errors for measurements done with a traveling microscope are: b-a about $7.900 \pm .005 \mathrm{~cm}( \pm .06 \%)$, B-A greater than $1.200 \pm .005 \mathrm{~cm}( \pm .4 \%)$ for second order line, or $0.600 \pm .005 \mathrm{~cm}$ or greater $( \pm .8 \%)$ for 1st order line. To minimize the error in B-A, especially 
in case where higher order reflections were unavailable the difference $A-a$ was used instead of $B-A$,

$$
\begin{aligned}
\text { since } & (B-A) / 2=(b-a) / 2-(A-a) \\
& (B-A)=(b-a)-2(A-a) \\
& d=(1-2(A-a) /(b-a))^{-1} d_{\text {Teflon }}
\end{aligned}
$$

which even for the first order diffraction line has an error smaller than $1 \%$.

It was observed that when two or more films were loaded in the camera and no provision was made to establish a true value of $\mathrm{R}$ for each film the errors in $\mathrm{d}$ for different films were in some cases higher than $2 \%$. 